\title{
Multiple domains in ARHGAP36 regulate PKA degradation and Gli activation
}

${ }^{1}$ Department of Chemical and Systems Biology, Stanford University School of Medicine, Stanford, CA

${ }^{2}$ Department of Microbiology and Immunology, Stanford University School of Medicine, Stanford, CA

${ }^{3}$ Department of Developmental Biology, Stanford University School of Medicine, Stanford, CA ${ }^{4}$ Department of Chemistry, Stanford University, Stanford, CA ${ }^{5}$ Present address: Brain Science and Advanced Technology Institute, Wuhan University of Science and Technology, Wuhan, China

Patricia R. Nano', Takamasa Kudo¹, Nancie A. Mooney², Jun $\mathrm{Ni}^{1,5}$, Janos Demeter ${ }^{2}$, Peter K. Jackson², and James K. Chen ${ }^{1,3,4, *}$

(3)
.

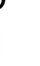

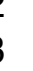
45 6

\footnotetext{
* Correspondence should be addressed to J.K.C. (jameschen@stanford.edu)
} 


\section{ABSTRACT}

ARHGAP36 is a Rho GTPase-activating protein (GAP) family member that contributes to

51 spinal cord development and tumorigenesis. This multidomain protein is composed of splicing-

52 dependent $\mathrm{N}$-terminal sequences, the GAP-like region, and a unique C-terminal domain, and an

$53 \mathrm{~N}$-terminal arginine-rich region has been shown to suppress protein kinase $\mathrm{A}(\mathrm{PKA})$ and activate

54 Gli transcription factors. To understand how these structural elements act in concert, we have

55 mapped the ARHGAP36 structure-activity landscape with domain- and amino-acid-level

56 resolution. ARHGAP36-mediated Gli activation can be repressed by N-terminal sequences that

57 regulate subcellular ARHGAP36 localization and PKA targeting. The GAP-like and C-terminal

58 domains counteract this autoinhibitory mechanism and promote ARHGAP36 trafficking to the

59 plasma membrane and primary cilium, respectively. The GAP-like domain may also conditionally

60 suppress the arginine-rich region, and it modulates ARHGAP36 binding to the prolyl

61 oligopeptidase-like protein PREPL and the E3 ubiquitin ligase PRAJA2. These domain-dependent

62 activities provide a potential means for tissue-specific ARHGAP36 functions.

63 


\section{INTRODUCTION}

Gli transcription factors (Gli1-3) are essential regulators of cell proliferation and

71 differentiation, controlling fate specification in the neural tube (Briscoe, et al., 2000; Stamataki, et

al., 2005) and limb bud (Hill, et al., 2009; te Welscher, et al., 2002) and the maintenance of granule

neuron precursors in the developing cerebellum (Lewis, et al., 2004; Wallace, 1999; Wechsler-

74

75

76

77

78

79

80

81

82

83

84

85

86

87

88

89

90

91

92

93

Reya, et al., 2001). Accordingly, misregulation of Gli activity can lead to uncontrolled cell growth, resulting in basal cell carcinoma, medulloblastoma, and other human cancers (Hui, et al., 2011).

Gli functions are primarily regulated by the Hedgehog $(\mathrm{Hh})$ pathway, and in the absence of $\mathrm{Hh}$ ligands, GLI2 and GLI3 are bound to the scaffolding protein Suppressor of Fused (SUFU) (Stone, et al., 1999; Wang, Chengbing, et al., 2010), which promotes their sequential phosphorylation by protein kinase $A(P K A)$, glycogen synthase kinase $\beta(G S K 3 \beta)$, and casein kinase 1 (CK1) (Pan, et al., 2006; Pan, et al., 2007; Tempe, et al., 2006; Wang, Baolin, et al., 2006). Proteasomal machinery is then recruited to the phosphorylated Gli proteins, resulting in GLI2 degradation and proteolytic conversion of GLI3 into a transcriptional repressor (Pan, et al., 2007).

Hh ligands suppress these intracellular processes, acting via the transmembrane receptors Patched1 $(\mathrm{PTCH} 1)$ and Smoothened (SMO). Through mechanisms that remain unclear, SMO promotes the dissociation of GLI2 and GLI3 from SUFU, uncoupling the transcription factors from proteasomal regulation and allowing the full-length proteins to become transcriptional activators (Humke, et al., 2010; Tukachinsky, et al., 2010). SMO activity is suppressed by PTCH1 (Murone, et al., 1999; Rohatgi, et al., 2007; Taipale, J., et al., 2002), which is in turn directly inhibited by Hh ligands (Incardona, et al., 2000; Stone, et al., 1996). Hh signaling therefore induces SMO activation and the expression of $\mathrm{Hh}$ target genes, including those that encode PTCH1 (Ågren, et al., 2004) and the constitutively active transcription factor GLI1 (Bai, et al., 2004; Dai, et al., 1999). The primary cilium serves a key center for these signaling events (Dorn, et al., 2012; Haycraft, et al., 2005; Kim, et al., 2009; May, et al., 2005; Rohatgi, et al., 2007; 
94 Wang, $Y$ u, et al., 2009; Wen, et al., 2010), and this cell-surface protrusion is required for both Gli 95 activator and repressor formation (Huangfu, et al., 2005; Liu, Aimin, et al., 2005). In addition to these signal transduction mechanisms, there is growing evidence for noncanonical Gli regulation in both normal physiology (Dennler, et al., 2007; Flora, et al., 2009; Riobó, et al., 2006) and in cancer (Beauchamp, et al., 2009; Dennler, et al., 2007; Elsawa, et al., 2011; Han, et al., 2015; Kasper, et al., 2006; Liu, Z., et al., 2014; Long, et al., 2014). Our laboratory previously established ARHGAP36 as a non-canonical Gli activator that acts in a SMOindependent manner (Rack, et al., 2014). We identified this Rho GTPase-activating protein (GAP) family member in a genome-scale screen for Hh pathway agonists (Rack, et al., 2014), and subsequent studies have uncovered an essential role for ARHGAP36 in the specification of lateral motor column neurons (Nam, et al., 2019; Rack, et al., 2014). Endogenous Arhgap36 transcription in the developing mouse spinal cord coincides with Hh pathway activation, and its overexpression leads to ectopic induction of the Hh target genes Ptch1 and Gli1 (Nam, et al., 2019). In addition, Arhgap36 expression has been found to correlate with SMO inhibitor resistance in Hh pathwaydriven murine medulloblastomas (Buonamici, et al., 2010; Rack, et al., 2014), and upregulating this Rho GAP family member in neural progenitor cells is sufficient to induce medulloblastomas in mice (Beckmann, et al., 2019). ARHGAP36 may promote tumor growth through multiple mechanisms, as elevated ARHGAP36 expression also has been associated with Hh pathwayindependent subtypes of medulloblastoma and neuroblastoma (Beckmann, et al., 2019; Lee, et al., 2019). biochemical and cellular mechanisms that regulate and transduce its activity are not well understood. The ARHGAP36 protein consists of unique $\mathrm{N}$ - and C-terminal domains and a central 117 region that is homologous to Rho GAPs. In addition, the five annotated isoforms of human 
ARHGAP36 isoforms can activate Gli proteins, with the gene product harboring the longest Nterminal domain (isoform 1) being the sole exception (Rack, et al., 2014). Isoform 1 is also the only ARHGAP36 protein that does not localize to the plasma membrane, and it instead adopts a perinuclear distribution (Müller, et al., 2020; Rack, et al., 2014). In addition, the shortest ARHGAP36 protein (isoform 3) accumulates in the primary cilium, whereas other ARHGAP36 isoforms cannot be detected in this signaling center under steady-state conditions (Rack, et al., 2014). More recently, it has been shown that an N-terminal arginine-rich motif conserved in all human ARHGAP36 isoforms can bind directly to catalytic subunits of PKA (PRKACA and PRKACB; henceforth referred to as PKA $\mathrm{Cat}_{\text {) }}$ (Eccles, et al., 2016). In the context of isoform 2, this motif mediates the degradation of $\mathrm{PKA}_{\text {cat }}$, and a 77-amino-acid $\mathrm{N}$-terminal fragment that includes this arginine-rich region has been shown to be necessary and sufficient for cellular $P K A_{c a t}$ depletion (Eccles, et al., 2016). These findings suggest a role for $\mathrm{N}$-terminal sequences in targeting ARHGAP36 to specific subcellular compartments and establish PKA anhibition as a potential basis for ARHGAP36-mediated Gli activation.

In comparison, the functions of the GAP-like and C-terminal domains in ARHGAP36 have not yet been elucidated. Rho GAP family members typically attenuate the function of Rho GTPases by stimulating GTP hydrolysis (Moon, 2003). However, the GAP-like region in ARHGAP36 lacks the "arginine finger" motif conserved in catalytically active homologs (Rack, et al., 2014; Scheffzek, et al., 1998), and ARHGAP36 has no effect on the activities of Rac1, Cdc42, and Rho A (Müller, et al., 2020). In addition, ARHGAP36 residues that are structurally equivalent to those previously associated with Rho GAP-catalyzed GTP hydrolysis are not required for ARHGAP36-mediated Gli activation (Rack, et al., 2014). Non-catalytic mechanisms have been

141 reported for several Rho GAP family members (Amin, et al., 2016; Faucherre, et al., 2003; Marchesi, et al., 2014), and it is possible that the GAP-like domain in ARHGAP36 similarly interacts with Rho GTPases or other signaling proteins in a stoichiometric manner. How the C- 
144 terminal domain might contribute to ARHGAP36 function is even more enigmatic since it lacks 145 sequence homology with other proteins. requires a deeper understanding of the relationship between ARHGAP36 structure and function.

148 Here we describe our systematic mapping of the ARHGAP36 structure-activity landscape using 149 individual ARHGAP36 isoforms, truncated variants, and a high-throughput mutagenesis screen.

150 Our findings demonstrate that ARHGAP36-dependent Gli activation and cellular PKA cat $_{\text {depletion }}$ 151 are separable activities and reveal isoform-specific differences in subcellular $\mathrm{PKA}_{\text {cat }}$ targeting. 152 While the ARHGAP36 N-terminal domain is necessary and sufficient for Gli activation, an N153 terminal region in isoform 2 (residues 1-105; N2 $1-105$ ) can inhibit this function and suppress protein 154 localization to the plasma membrane. This autoinhibitory mechanism is counteracted by the GAP155 like and C-terminal domains, which promote ARHGAP36 recruitment to the plasma membrane 156 and primary cilium, respectively. Finally, we have discovered several residues within the GAP157 like domain that are necessary for Gli activation by full-length ARHGAP36 isoforms. These 158 residues are predicted to cluster within the GAP-like domain structure, at a site distal to the Rho 159 GTPase-binding pocket, and they are required for ARHGAP36 recruitment to the plasma membrane. We have also leveraged these mutants to discover factors that bind specifically to the 161 wild-type protein, identifying potential mediators of ARHGAP36 function. Taken together, our work 162 supports a model in which ARHGAP36 activity state, subcellular localization, and effector binding 163 are regulated by structural elements distributed throughout protein. In combination with the 164 differential expression of ARHGAP36 isoforms, such mechanisms could allow ARHGAP36 to

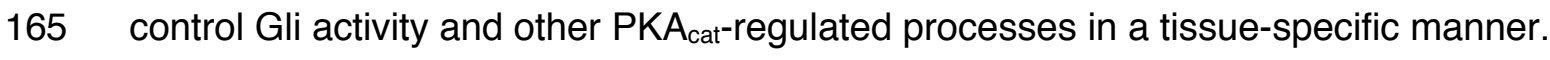




\section{RESULTS}

\section{Gli activation and cellular PKA $A_{c a t}$ depletion are separable ARHGAP36 functions}

To explore the relationship between ARHGAP36-mediated Gli activation and PKA $\mathrm{A}_{\text {cat }}$ degradation, we measured the effect of each human ARHGAP36 isoform on both cellular processes. Individual isoforms were retrovirally transduced into NIH-3T3 mouse fibroblasts, a commonly used line for studying $\mathrm{Hh}$ signal transduction (Taipale, J, et al., 2000) that also exhibits ARHGAP36 responsiveness (Eccles, et al., 2016; Rack, et al., 2014). The resulting levels of Gli1 mRNA and $\mathrm{PKA}_{\text {cat }}$ protein were assessed by qRT-PCR and western blot, respectively.

Overexpression of isoforms 2, 4, or 5 was sufficient to activate Gli and deplete the cells of PKA $\mathrm{A}_{\text {cat }}$, while isoform 1 exhibited neither activity (Figure 1B). In contrast, transduction of isoform 3 induced Gli1 expression without reducing cellular $\mathrm{PKA}_{\text {cat }}$ levels to a discernable extent. These results indicate that total $\mathrm{PKA}_{\text {cat }}$ depletion is not required for ARHGAP36-mediated Gli activation, raising the possibility that ARHGAP36 regulates Gli proteins by targeting a specific subcellular pool of $\mathrm{PKA}_{\text {cat }}$ and/or through PKA-independent mechanisms.

We investigated these two models by further comparing the activities of ARHGAP36 isoform 3 with those of isoform 2. Using immunofluorescence microscopy, we observed that isoform 2 globally depleted $\mathrm{PKA}_{\text {cat }}$ in NIH-3T3 cells, corroborating our western blot analyses (Figure 1C). In contrast, isoform 3 reduced $\mathrm{PKA}_{\text {cat }}$ pools predominantly in the Golgi. These findings are consistent with the accumulation of isoform 3 in the primary cilium (Rack, et al., 2014), as the cilium base communicates directly with the Golgi through vesicular trafficking (Pedersen, et al., 2016). We next examined how the activities of ARHGAP36 isoforms 2 and 3 are affected by forskolin, an adenylate cyclase agonist that increases cAMP levels and PKA $\mathrm{Aat}_{\text {activity. We }}$ transduced the ARHGAP36 constructs into NIH-3T3 fibroblasts stably expressing a Glidependent firefly luciferase reporter (SHH-LIGHT2 cells) (Taipale, J, et al., 2000) and treated the cells with the PKA $\mathrm{Pat}_{\text {at }}$ activator. Although the isoform 2-expressing cells exhibited almost two-fold 
bioRxiv preprint doi: https://doi.org/10.1101/2020.05.14.094961; this version posted May 24, 2020. The copyright holder for this preprint (which was not certified by peer review) is the author/funder. All rights reserved. No reuse allowed without permission.
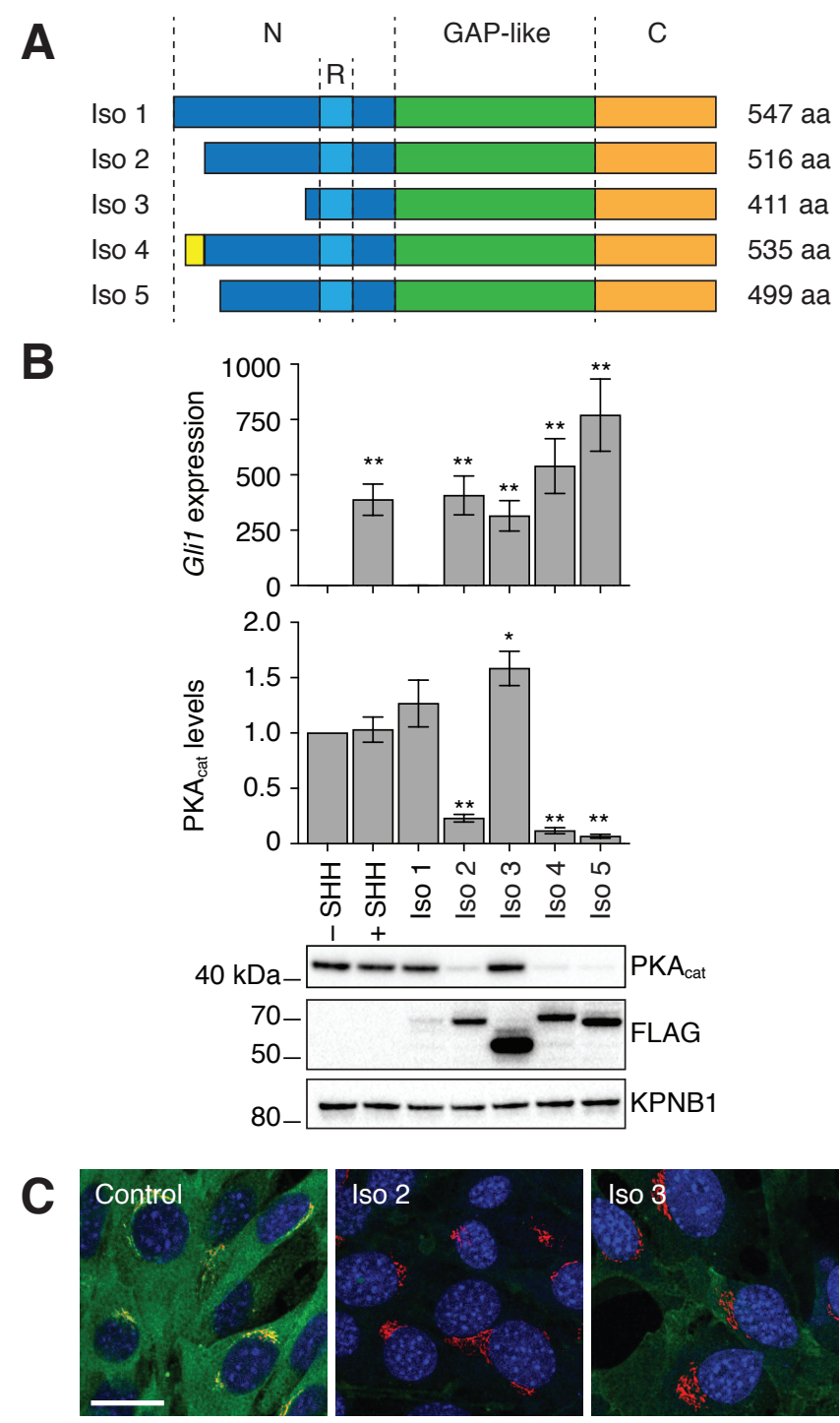

$\mathrm{PKA}_{\text {cat }}$ GM130 DAPI
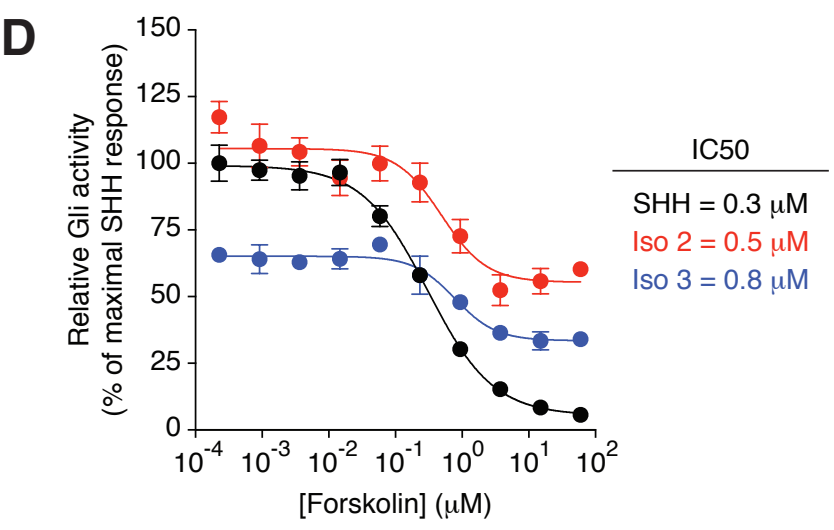

Figure 1. ARHGAP36 isoforms differentially induce PKA cat $_{\text {che }}$ deplion and Gli activation. (A) Domain architecture of the five human ARHGAP36 isoforms, with the N-terminal domain $(\mathrm{N})$ shown in dark blue, arginine-rich motif (R) in light blue, GAP-like domain in green, and C-terminal domain (C) in orange. The yellow region indicates an amino acid sequence unique to isoform 4 . (B) Gli1

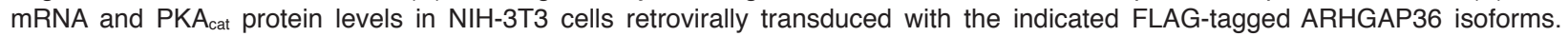
Uninfected cells treated with or without Sonic Hedgehog $(\mathrm{SHH})$ ligand were included as positive and negative controls, respectively. Data are the average fold change relative to the negative control for three biological replicates \pm s.e.m. Single and double asterisks indicate $P<0.05$ and $P<0.01$, respectively. A representative western blot for each condition is also shown. (C) PKA cat localization in NIH-3T3 cells transduced with FLAG-tagged ARHGAP36 isoform 2 or 3 . Representative immunofluorescence micrographs are shown with staining for PKA cat $_{1}$ GM130 (cis-Golgi), and DAPI (nucleus). Scale bar: $20 \mu \mathrm{m}$. (D) Forskolin dose-response curves for SHH-LIGHT2 cells stimulated with SHH or transduced with FLAG-tagged ARHGAP36 isoform 2 or 3. Data are the average Gli reporter activities for at least three biological replicates \pm s.e.m., normalized to the maximum response in SHH-treated cells. 
192 higher Gli reporter activity than those expressing isoform 3, forskolin inhibited Gli function in both

193 lines with comparable IC50s (Figure 1D). Maximal doses of the PKA at activator also suppressed 194 about $50 \%$ of the Gli reporter activity induced by each isoform. Together, these results demonstrate that $\mathrm{N}$-terminal sequences in ARHGAP36 can regulate its ability to target $\mathrm{PKA}_{\text {cat }}$ in specific subcellular compartments and raise the possibility that ARHGAP36 activates Gli proteins

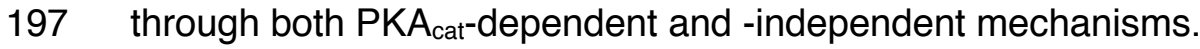

ARHGAP36 is autoinhibited by an N-terminal region that is counteracted by the GAP-like and Cterminal domains

We continued to investigate functional differences between ARHGAP36 isoforms 2 and 3 by determining the activities of various truncation mutants (Figure $2 \mathrm{~A}$ ). By retrovirally expressing these constructs in NIH-3T3 cells, we observed that the N-terminal domain of ARHGAP36 isoform 2 (residues 1-194; N2) is necessary and sufficient for its effects on Gli and PKA $\mathrm{A}_{\text {cat }}$ (Figure 2B) corroborating previous reports (Eccles, et al., 2016). However, N2 was less effective at activating Gli1 expression than the N-terminal domain of isoform 3 (N3), even though N2 could induce $\mathrm{PKA}_{\text {cat }}$ degradation. N2 was also markedly less active than full-length isoform 2. In contrast, N3 and full-length isoform 3 could induce Gli1 expression to similar extents (Figure 2B). These results indicate that the $\mathrm{N} 2$ region absent in isoform 3 (residues 1-105; N2 ${ }_{1-105}$ ) represses

210 the Gli-activating function of the remaining N-terminal domain. Moreover, our findings suggest 211 that ARHGAP36 sequences in the GAP-like and/or C-terminal domains can influence $\mathrm{N} 2_{1-105}$ 212 function.

To discern how the GAP-like and C-terminal domains contribute to ARHGAP36 function,

214 we examined the activities of N2-GAP and N3-GAP constructs in NIH-3T3 cells. N2-GAP was 215 moderately more active than N2 with respect to Gli1 expression, but it was still much less active 216 than full-length isoform 2 (Figure 2B). N3-GAP activity was comparable to that of N3 and full- 
bioRxiv preprint doi: https://doi.org/10.1101/2020.05.14.094961; this version posted May 24, 2020. The copyright holder for this preprint (which was not certified by peer review) is the author/funder. All rights reserved. No reuse allowed without permission.

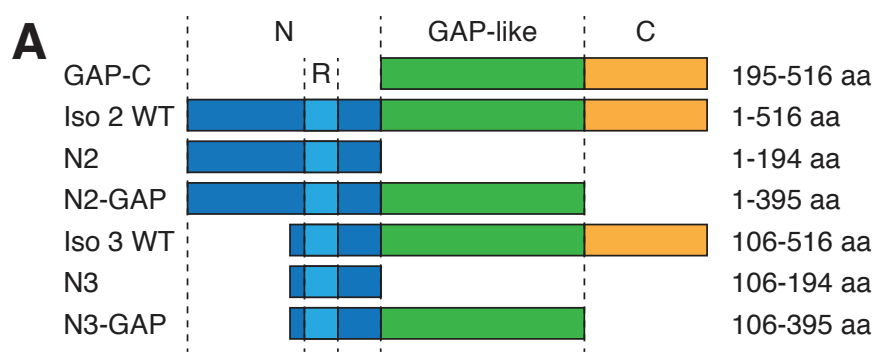

B
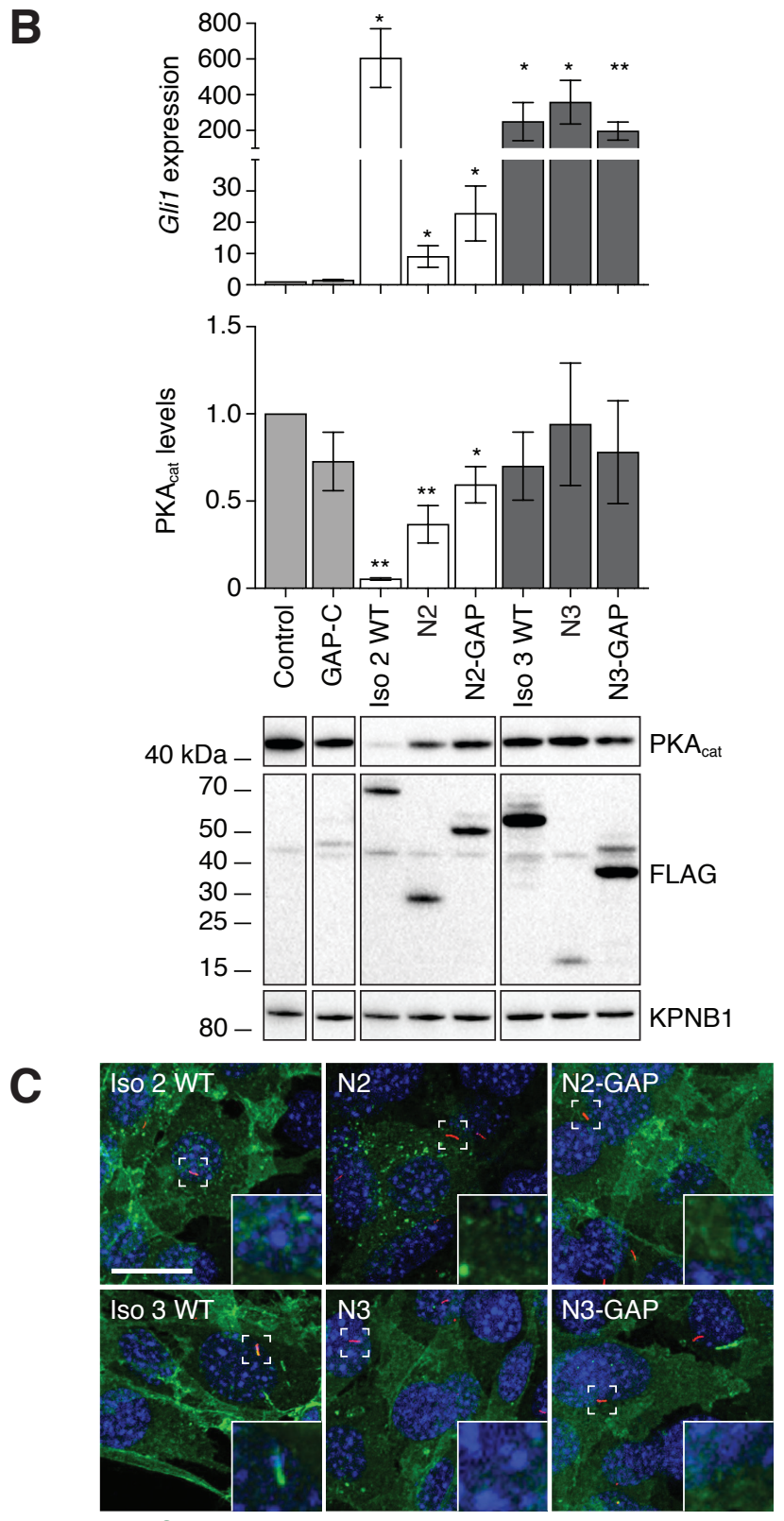

FLAG ARL13B DAPI

Figure 2. N-terminal, GAP-like, and C-terminal domains have opposing effects on ARHGAP36 function. (A) Schematic representation of ARHGAP36 isoform 2 or 3 truncation mutants. Residue numbers are based on the amino acid sequence of

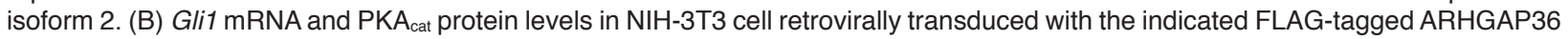
truncation mutants. Data are the average fold change relative to uninfected cells for three biological replicates \pm s.e.m. Single and double asterisks indicate $P<0.05$ and $P<0.01$, respectively. A representative western blot for each condition is also shown (lanes from the same blot image have been cropped and re-ordered for clarity). (C) Subcellular distributions of the indicated FLAG-tagged ARHGAP36 constructs in NIH-3T3 cells. Representative immunofluorescence micrographs are shown with staining for FLAG, ARL13B (primary cilium) and DAPI (nucleus). Insets highlight ciliated regions in the dashed boxes. Scale bar: $20 \mu \mathrm{m}$. Images were processed to establish comparable maximum pixel intensities in order to highlight differences in localization. 
217 length isoform 3. Thus, both the GAP-like and C-terminal domains can counteract the 218 autoinhibitory function of $\mathrm{N} 2_{1-105}$, with the $\mathrm{C}$-terminal region playing a particularly important role.

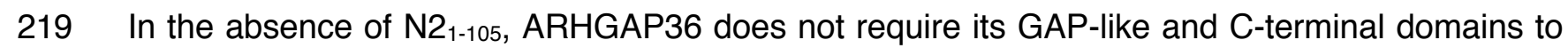
220 achieve high levels of Gli activity.

We next sought to determine how the $\mathrm{N} 2_{1-105}$ region, GAP-like domain, and C-terminal domain regulate ARHGAP36 function. The differing subcellular distributions of full-length isoforms 2 and 3 indicate that the N2 ${ }_{1-105}$ region influences ARHGAP36 trafficking (Rack, et al., 2014), and we therefore examined the localizations of the truncation mutants. N2 accumulated in both punctate structures and the plasma membrane, whereas N2-GAP was robustly recruited to the latter (Figure 2C). In comparison, both N3 and N3-GAP predominantly associated with the plasma membrane, and unlike the full-length isoform 3, neither construct accumulated in the primary cilium.

These domain-dependent changes in protein localization indicate that $\mathrm{N} 2{ }_{1-105}$ impedes and the GAP-like domain facilitates ARHGAP36 translocation to the plasma membrane. In principle, these opposing activities could involve direct interactions between the two regions in ARHGAP36 or parallel functions involving other factors. In addition, the C-terminal domain acts independently of the $\mathrm{N} 2_{1-105}$ region to promote ciliary accumulation of ARHGAP36. The disparate roles of these domains in ARHGAP36 trafficking correlate with their divergent effects on Gli activation, providing further evidence that ARHGAP36 targets specific subcellular compartments to regulate Gli 236 proteins. 
bioRxiv preprint doi: https://doi.org/10.1101/2020.05.14.094961; this version posted May 24, 2020. The copyright holder for this preprint (which was not certified by peer review) is the author/funder. All rights reserved. No reuse allowed without permission.
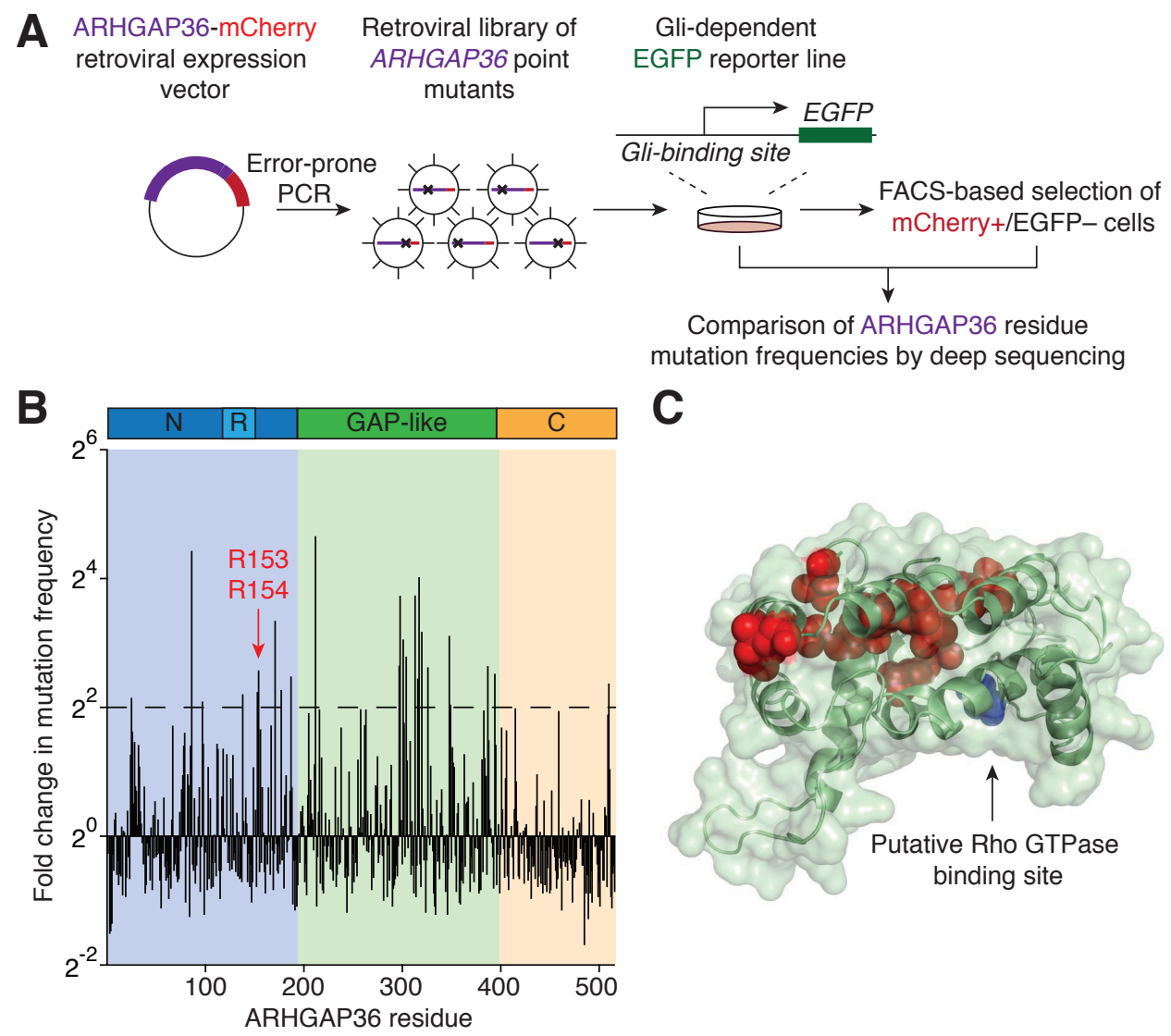

C

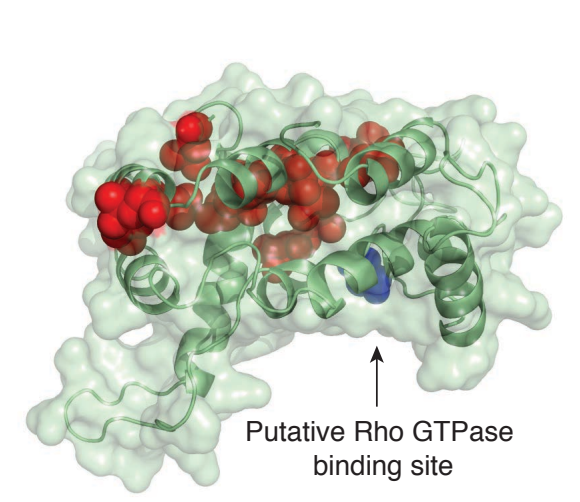

D

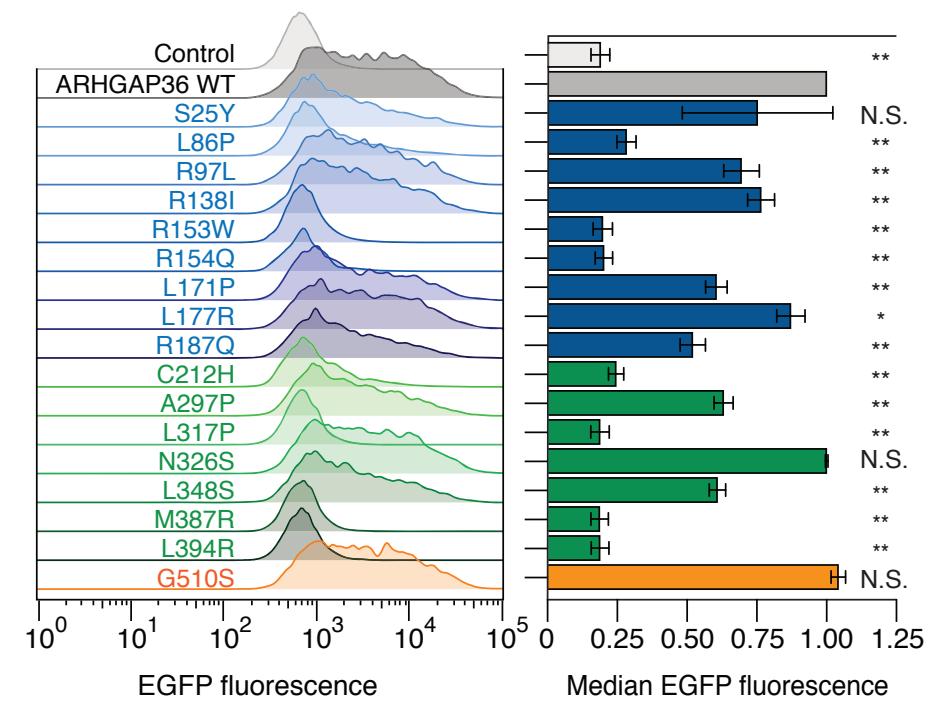

Figure 3. Identification of essential residues within the $\mathrm{N}$-terminal and GAP-like domains. (A) Schematic representation of the high-throughput mutagenesis screen used to identify individual residues that contribute to ARHGAP36 function. (B) Histogram depicting the fold change in mutation frequency between pre- and post-selected population for each residue in ARHGAP36. (C) Homology model of the ARHGAP36 GAP-like domain structure based on the crystal structure of 32 -chimaerin (PDB ID: 1xa6). Residues with $>4$-fold change in mutation frequency are shown in red, and the site (Thr227) that is structurally equivalent to the arginine finger is shown in blue. (D) Activities of selected ARHGAP36 variants in SHH-EGFP cells, as assessed by flow cytometry-based measurements of Gli reporter fluorescence. The distributions (left) and relative medians (right) of EGFP fluorescence are shown for each ARHGAP36 construct. Data are the average fold change in median EGFP fluorescence relative to that of cells expressing wild-type ARHGAP36 for three biological replicates \pm s.e.m. Single and double asterisks indicate $P<0.05$ and $P<0.01$, respectively. 
242 variants C-terminally fused to mCherry, obtaining a collection of approximately 100,000 single 243 point mutants (27\% of all library constructs and 22 -fold theoretical coverage of the 4,374 possible 244 variants). The library was retrovirally transduced into NIH-3T3 fibroblasts expressing a Glidependent green-fluorescent reporter (SHH-EGFP cells) (Hyman, et al., 2009) using a multiplicity of infection (MOI) of 0.3 to maximize the number of cells with single integration events. Cells expressing full-length ARHGAP36 proteins were then isolated by fluorescence-activated cell sorting (FACS) according to their mCherry fluorescence. We next cultured these cells under Hh signaling-competent conditions to allow active ARHGAP36 mutants to induce Gli-dependent EGFP expression, after which mCherry+/EGFP- cells were isolated by FACS. To ensure that these ARHGAP36-expressing cells still harbored a functional EGFP reporter, they were 252 subsequently cultured with the SMO agonist SAG (Chen, et al., 2002). The resulting mCherry+/EGFP+ cells were obtained by FACS, yielding a population of cells expressing putative, inactive ARHGAP36-mCherry mutants.

To identify inactivating point mutations, we used genomic PCR and deep sequencing to compare the mutation frequency of each amino acid position in the pre- and post-selection populations. This analysis revealed several residues that could be required for Gli activation, including two N-terminal arginines (R153 and R154) that were previously shown to be required

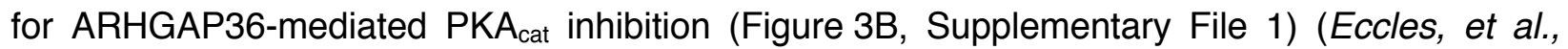
2016). The majority of these putative essential residues were located in the GAP-like region, and structure homology modeling of this domain predicts that these amino acids cluster together at a site that is distal to the predicted Rho GTPase binding pocket (Figure 3C). We then used flow cytometry-based assays to validate a subset of these ARHGAP36 variants, prioritizing residues

264 that were mutated $>$ 4-fold more frequently in the inactive mutant pool. In these experiments, 17 265 individual ARHGAP36 mutants were retrovirally transduced into cells at an $\mathrm{MOI}$ of 0.3 to 
point mutations significantly decreased ARHGAP36 activity in SHH-EGFP reporter cells

268 (Figure 3D).

GAP-like domain mutations disrupt ARHGAP36 recruitment to the plasma membrane

Guided by the results of our mutagenesis screen, we investigated how individual point mutations might regulate specific aspects of ARHGAP36 function. Our studies focused on the three residues with the greatest fold change in pre- and post-selection mutation frequencies:

274 N-terminal domain residue L86 and two sites within the GAP-like domain, C212 and L317

275 (Figure 4A). We first examined how mutations at these sites affect the ability of ARHGAP36 276 isoform 2 to induce Gli1 expression and PKA cat degradation in NIH-3T3 cells. All three variants

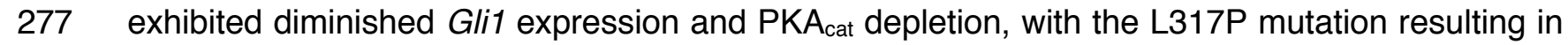
278 complete loss of both ARHGAP36-dependent activities (Figure 4B). We next determined the 279 effects of these mutations on the subcellular localization of ARHGAP36 isoform 2. The L86P 280 variant retained the ability to localize to the plasma membrane, but both mutations in the GAP281 like domain rendered ARHGAP36 cytosolic (Figure 4C). To further elaborate the contributions of individual residues to ARHGAP36 trafficking, we also assessed the localization of other ARHGAP36 point mutants that were inactive in SHH-EGFP cells (see Figure 3D). The R153W and R154Q variants accumulated in the plasma membrane, and the GAP-like domain point mutants M387R and L394R remained primarily in the cytosol 286 (Figure 4 - figure supplement 1). Together, these results provide further evidence that the GAP287 like domain recruits ARHGAP36 to the cell membrane, counteracting the function of N2 $1-105$. We 288 then investigated whether plasma membrane recruitment by the GAP-like domain requires $\mathrm{N} 2{ }_{1-105}$ 289 or the C-terminal domain, both of which affect ARHGAP36 localization. As in the full-length 290 isoform 2, C212Y and L317P mutations impeded the ability of N2-GAP to localize to the plasma 291 membrane and activate Gli proteins (Figure 5A-B). Structurally equivalent mutations (C107Y and 
bioRxiv preprint doi: https://doi.org/10.1101/2020.05.14.094961; this version posted May 24, 2020. The copyright holder for this preprint (which was not certified by peer review) is the author/funder. All rights reserved. No reuse allowed without permission.

A

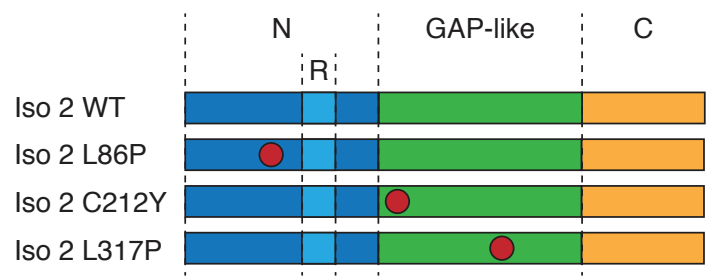

B

C 1 Iso 2 WT
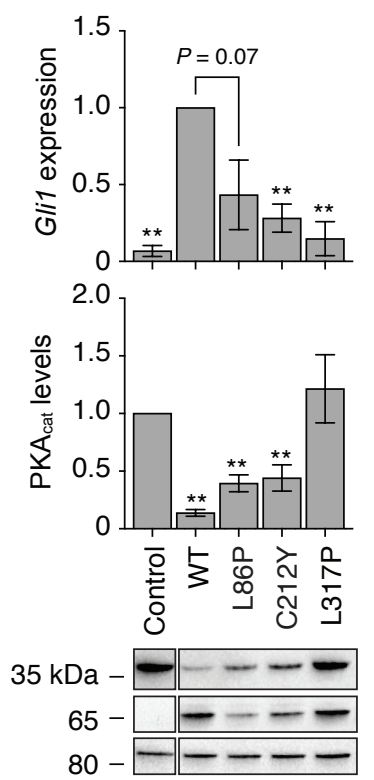

PKA $_{\text {cat }}$

FLAG

KPNB1

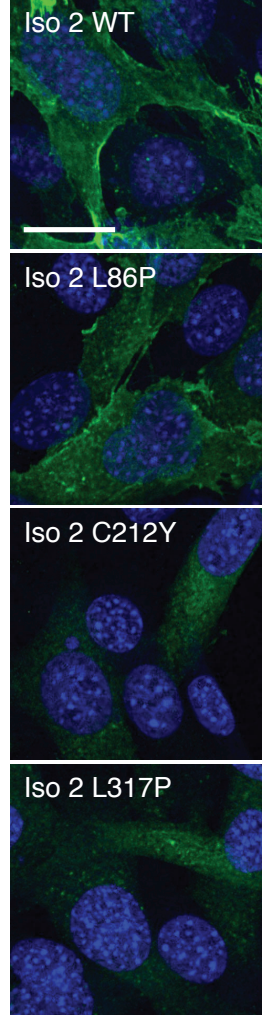

FLAG DAPI

Figure 4. Point mutations in the GAP-like domain cause ARHGAP36 mislocalization. (A) Schematic representation of ARHGAP36 isoform 2 point mutants. (B) Gli1 mRNA and PKA cat protein levels in NIH-3T3 cells retrovirally transduced with the indicated FLAG-tagged ARHGAP36 constructs. Uninfected cells were used as controls. Data are the average fold change relative to cells expressing wild-type ARHGAP36 (Gli1 expression) or to untreated cells (PKA cat levels) for three biological replicates \pm s.e.m. Single and double asterisks indicate $P<0.05$ and $P<0.01$, respectively. A representative western blot for each condition is also shown (lanes from the same blot image have been cropped and re-ordered for clarity). (C) Subcellular distributions of the indicated FLAG-tagged ARHGAP36 constructs in NIH-3T3 cells. Representative immunofluorescence micrographs are shown with staining for FLAG and DAPI (nucleus). Scale bar: $20 \mu \mathrm{m}$. 
bioRxiv preprint doi: https://doi.org/10.1101/2020.05.14.094961; this version posted May 24, 2020. The copyright holder for this preprint (which was not certified by peer review) is the author/funder. All rights reserved. No reuse allowed without permission.

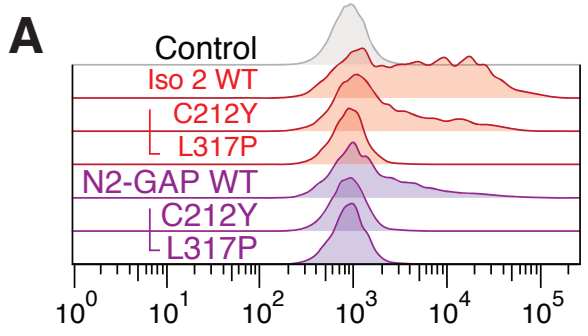

EGFP fluorescence

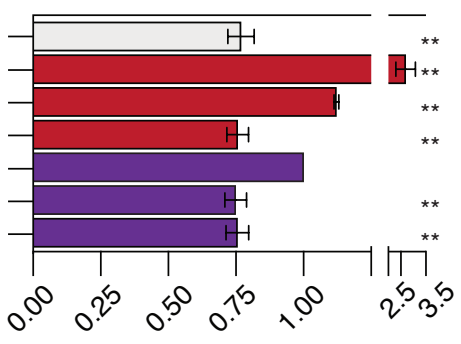

Median EGFP fluorescence

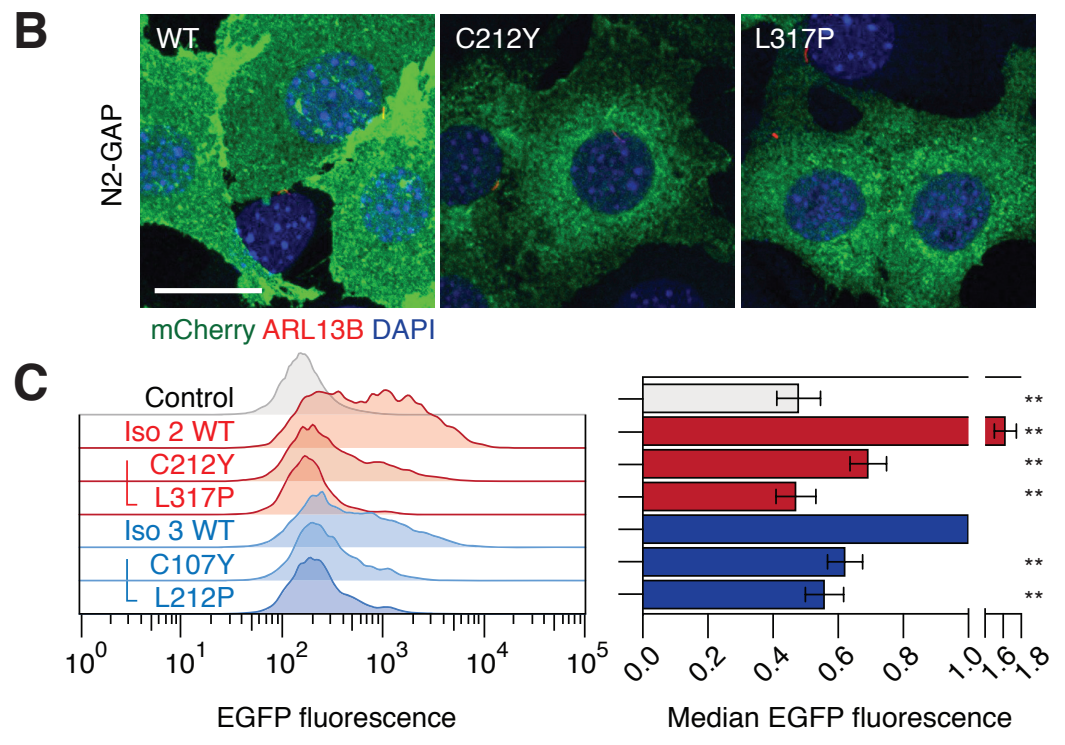

D
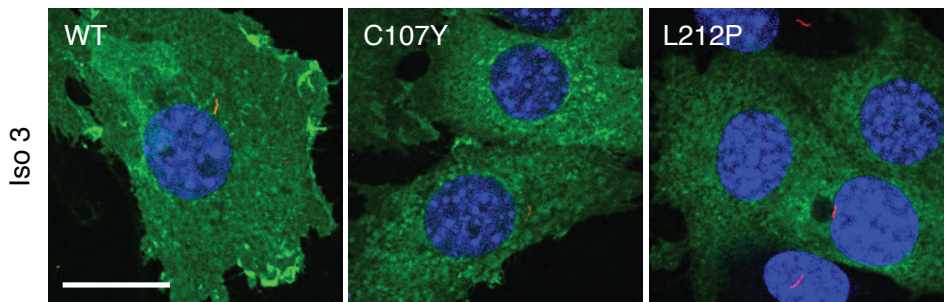

mCherry ARL13B DAPI

Figure 5. Point mutations in the GAP-like domain act independently of N21-105 and the C-terminal domain. (A and C) Effects of GAP-like domain point mutations in N2-GAP- or isoform 3-mediated Gli activation, as assessed in the flow cytometry-based SHH-EGFP assay. The distributions (left) and relative medians (right) of EGFP fluorescence are shown for each ARHGAP36 construct. Data are the average fold change in median EGFP fluorescence relative to that of cells expressing unmutated N2-GAP or isoform 3 for three biological replicates \pm s.e.m. Double asterisks indicate $P<0.01$. (B and $D)$ Subcellular distributions of the indicated mCherry-tagged ARHGAP36 constructs in SHH-EGFP cells. Representative immunofluorescence micrographs are shown with staining for mCherry, ARL13B (primary cilium), and DAPI (nucleus). Scale bars: $20 \mu \mathrm{m}$. 
L212P) in ARHGAP36 isoform 3, which lacks the $\mathrm{N} 2{ }_{1-105}$ region, also rendered the protein cytosolic and attenuated Gli activation (Figure 5C-D). Thus, the two GAP-like domain mutations disrupt ARHGAP36 function independently of $\mathrm{N}_{1-105}$ and the C-terminal domain.

GAP-like domain mutations alter the ARHGAP36 interactome

In addition to uncovering functional roles for specific ARHGAP36 structures, our collection

of inactive variants provided a means for identifying binding proteins that could participate in

ARHGAP36-mediated Gli activation. Toward this goal, we compared the interactomes of wild-

type and L317P ARHGAP36 isoform 2 by retrovirally transducing NIH-3T3 cells with vectors

encoding each ARHGAP36 construct fused to a C-terminal LAP tag (S-peptide-PreScission

protease site-EGFP) (Ding, et al., 2016; Hsu, et al., 2019; Kanie, et al., 2017; Li, Bin, et al., 2017;

Torres, et al., 2009; Wright, et al., 2011) (Figure 6A). To avoid total PKA cat depletion by wild-type

ARHGAP36 in these studies, we also limited the cells to a 4-hour incubation in retroviral medium and a subsequent 20-hour growth phase. The fibroblasts were then lysed, and each ARHGAP36 construct and its interacting proteins were isolated by tandem affinity purification and proteolytically digested as previously described (Ding, et al., 2016; Hsu, et al., 2019; Kanie, et al., 2017; Li, Bin, et al., 2017; Torres, et al., 2009; Wright, et al., 2011). The resulting peptides were sequenced and quantified using tandem mass spectrometry, and spectral counts were normalized

to account for variabilities in protein size, LAP tag purification efficiency, and ARHGAP36

311 expression. Using this approach, we identified 566 putative interactors for wild-type and/or L317P

312 ARHGAP36 that were observed across three biological replicates (Figure 6B, Supplementary

313 File 2). $\mathrm{PKA}_{\text {cat }}$ subunits were the only canonical Hh pathway regulators detected in these pulldown

314 experiments, and they interacted with wild-type and L317P ARHGAP36 to similar extents

315 (Figure 6B, Figure 6 - figure supplement 1). Eleven proteins preferentially bound to wild-type 
bioRxiv preprint doi: https://doi.org/10.1101/2020.05.14.094961; this version posted May 24, 2020. The copyright holder for this preprint (which was not certified by peer review) is the author/funder. All rights reserved. No reuse allowed without permission.
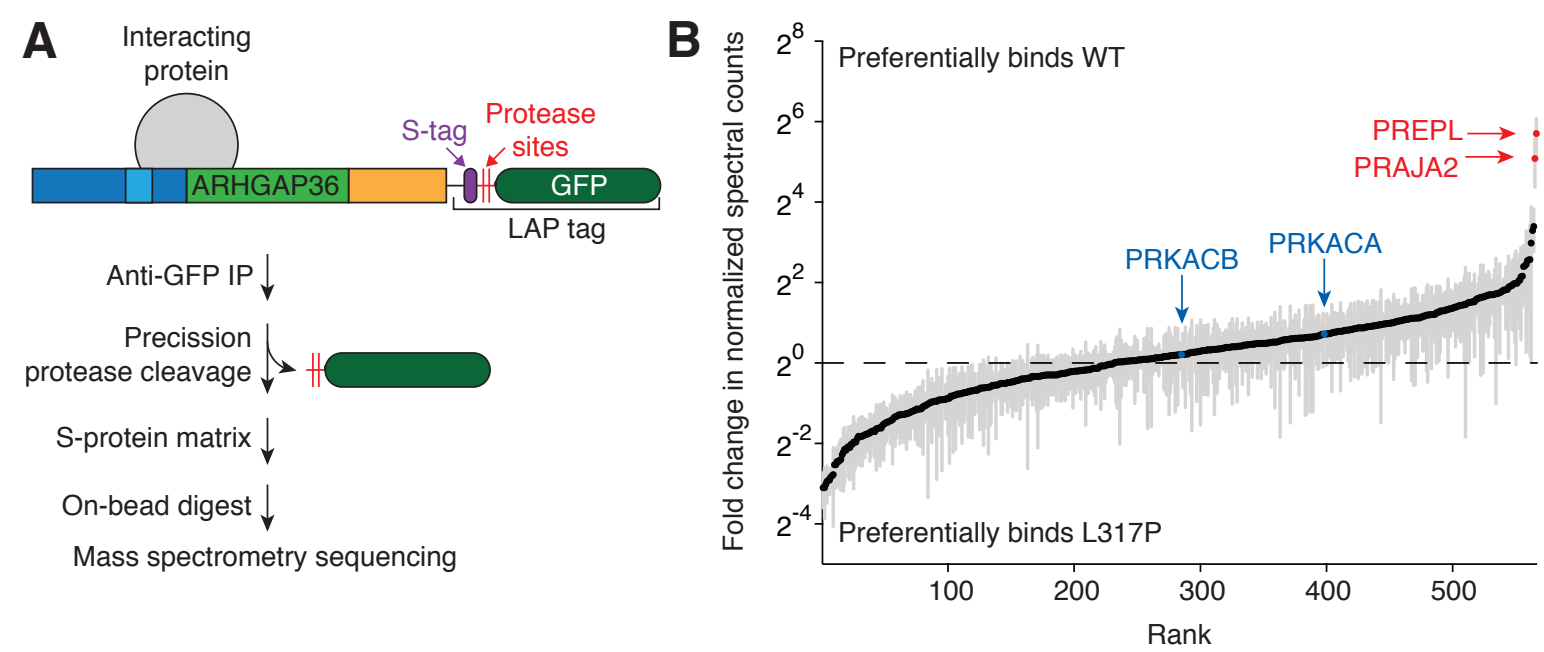

Figure 6. PREPL and PRAJA2 interact with ARHGAP36 in a GAP-like domain-dependent manner. (A) Schematic representation of the tandem affinity purification workflow for identifying ARHGAP36-binding proteins. (B) ARHGAP36 interactors ranked in order of their relative binding to the wild-type versus L317P proteins. Fold changes in normalized spectral counts represent the average values for three biological replicates \pm s.e.m., shown as grey bars. 
317 ligase PRAJA2 exhibiting the greatest selectivity (52- and 35-fold, respectively). PREPL displays

318 high sequence homology to a family of serine hydrolases, though its physiological substrates and 319 functions remain largely unknown (Jaeken, et al., 2006; Radhakrishnan, et al., 2013; Szeltner, et al., 2005). PRAJA2 has been shown to increase PKA activity by promoting the ubiquitination and degradation of regulatory $\mathrm{PKA}\left(\mathrm{PKA}_{\text {reg }}\right)$ subunits, a function that is enhanced by $\mathrm{PKA}_{\text {cat }}$ phosphorylation as part of a positive-feedback mechanism (Lignitto, et al., 2011). comparative interactome analyses therefore corroborate the results of these prior investigations and suggest that ARHGAP36-dependent Gli activation involves PREPL and PRAJA2 functions.

\section{DISCUSSION}

By systematically exploring the ARHGAP36 structure-activity landscape, we have identified key functional elements throughout this multidomain protein. Previous investigations identified an $\mathrm{N}$-terminal arginine-rich region that is necessary and sufficient for $\mathrm{PKA}_{\text {cat }}$ degradation signaling. Isoform-specific differences have also implicated N-terminal sequences in ARHGAP36 trafficking (Rack, et al., 2014). Our studies provide further evidence for these mechanisms of ARHGAP36 action, uncover a new functional module within the $\mathrm{N}$-terminal domain, and demonstrate important regulatory roles for the GAP-like and C-terminal domains (Figure 7).

Among our key findings is the discovery of an $\mathrm{N}$-terminal autoinhibitory region that is 339 present in isoforms 1 and 2. In the context of isoform 2, this sequence (N2 $\left.{ }_{1-105}\right)$ represses Gli1 340 activation by the $\mathrm{N}$-terminal domain and impedes its recruitment to the plasma membrane. $\mathrm{N} 2_{1-105}$ 341 likely regulates these activities through distinct mechanisms, since the L86P mutation in this 
bioRxiv preprint doi: https://doi.org/10.1101/2020.05.14.094961; this version posted May 24, 2020. The copyright holder for this preprint (which was not certified by peer review) is the author/funder. All rights reserved. No reuse allowed without permission.
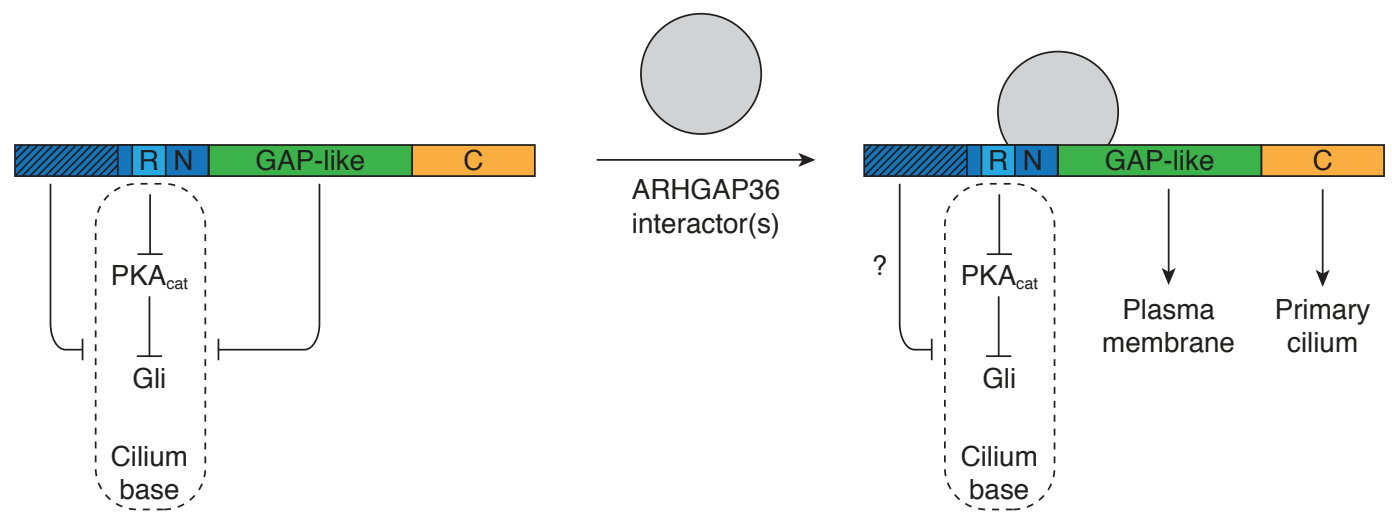

Figure 7. A regulatory model for ARHGAP36 function. Schematic representation of the structural elements that regulate ARHGAP36 localization and its ability to target Gli-regulating pools of PKA $A_{\text {cat }}$ in the primary cilium. N-terminal regulatory regions that vary between ARHGAP36 isoforms are depicted with hash marks. 
342 region diminishes the ability of isoform 2 to modulate $P K A_{c a t} / G$ li signaling without disrupting

343 membrane localization. ARHGAP36 isoform 3, which lacks the region corresponding to N2 ${ }_{1-105}$,

344 not only traffics to the plasma membrane but also accumulates in the primary cilium. The ciliary

345 localization of isoform 3 coincides with its ability to selectively degrade Golgi-localized PKA

346 likely due to vesicular transport between the cilium base and the Golgi (Pedersen, et al., 2016).

347 Our results suggest that the Gli-regulating pool of $\mathrm{PKA}_{\mathrm{cat}}$ traffics through these organelles, 348 corroborating previous reports that $\mathrm{Hh}$ signaling regulates PKA $\mathrm{Aat}_{\mathrm{cat}}$ activity in the basal body (Barzi,

349 et al., 2009; Tuson, et al., 2011; Zhang, et al., 2019). This mechanism of ARHGAP36-mediated

350 Gli activation parallels the actions of other Hh pathway regulators that modulate ciliary PKA

351 activity, such as adenylate cyclases 5 and 6 and the G-protein coupled receptor GPR161

352 (Chávez, et al., 2015; Garcia-Gonzalo, et al., 2015; Moore, et al., 2016; Mukhopadhyay, et al.,

353 2013; Vuolo, et al., 2015). ARHGAP36 might concurrently modulate Gli function via PKA-

354 independent mechanisms, since forskolin can only inhibit ARHGAP36-induced Gli activity by

$35550 \%$, even when maximal compound doses and different levels of Gli activation are employed.

Although the ARHGAP36 N-terminal domain alone can target $\mathrm{PKA}_{\text {cat }}$ for degradation and

harbors regulatory elements, its function is further modulated by other structures in the full-length

protein. The C-terminal domain strongly counteracts the autoinhibitory activity of $\mathrm{N} 2{ }_{1-105}$, as does

the GAP-like region to a more moderate extent. These regulatory actions likely involve protein

trafficking since the GAP-like and C-terminal domains promote ARHGAP36 recruitment to the

361 plasma membrane and primary cilium, respectively. However, we cannot rule out the possibility

362 that the two domains influence $\mathrm{N} 2_{1-105}$ function through additional mechanisms. Interestingly, the

363 ability of point mutations in the GAP-like domain to abrogate Gli activation by isoforms 2 and 3

364 contrasts the sufficiency of $\mathrm{N} 2$ and N3 for this process. The mutations also render these

365 ARHGAP36 proteins cytosolic. These differences could be explained if the unbound GAP-like 


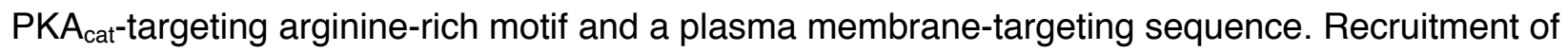
specific cellular factors to the GAP-like domain could then modulate this interaction and possibly mediate other ARHGAP36 activities (see Figure 7).

Which interacting proteins might regulate or transduce ARHGAP36 function remains to be determined, but our investigations provide valuable leads. By comparing the interactomes of wildtype ARHGAP36 and an inactive mutant, we have identified several proteins that bind to ARHGAP36 in a GAP-like domain-dependent manner. Although previous studies have shown that ARHGAP36 can co-immunoprecipitate with PTCH1 (Zhang, et al., 2019) and SUFU (Rack, et al., 2014) when they are overexpressed in cells, we do not observe analogous interactions with the endogenous Hh signaling proteins. Rho GTPases were also notably absent from the ARHGAP36 pulldowns, suggesting that ARHGAP36 interacts transiently with these signaling proteins or prefers other binding partners. In contrast, $\mathrm{PKA}_{\text {cat }}$ associated with both wild-type and L317P ARHGAP36 with comparable efficacies, indicating that the GAP-like domain mutation abrogates $\mathrm{PKA}_{\text {cat }}$ degradation without compromising its binding. Among the ARHGAP36 interactors discovered in our studies, PREPL and PRAJA2 emerged as the two most sensitive to the L317P mutation in the GAP-like domain. Both proteins were candidates in previous ARHGAP36 interactome datasets (Huttlin, et al., 2017; Müller, et al., 2020), but the functional relevance of these factors and other ARHGAP36-binding proteins has yet to be determined. By comparing the wild-type and mutant ARHGAP36 interactomes, we can provide a functional context for these binding proteins, implicating PREPL and PRAJA2 in ARHGAP36-dependent Gli activation.

In principle, the GAP-like domain could bind directly to PREPL or PRAJA2, or it could 389 allosterically regulate their interactions with other regions in the ARHGAP36 protein. PREPL is 390 highly homologous to the prolyl oligopeptidase (PREP) family of serine hydrolases, but its 
392 peptidic substrates could include factors that regulate PKA at or Gli activity. Alternatively, there is

393 evidence that PREP enzymes can modulate the metabolism of phosphoinositides (Schulz, et al., 394 2002; Williams, et al., 1999), a family of lipids with reported roles in the formation of Golgi-derived vesicles (Heldwein, et al., 2004; Wang, Ying Jie, et al., 2003) and the ciliary trafficking of Hh pathway regulators (Chávez, et al., 2015; Garcia-Gonzalo, et al., 2015). The interaction of ARHGAP36 with PRAJA2 is seemingly paradoxical since PRAJA2 has well-established roles in

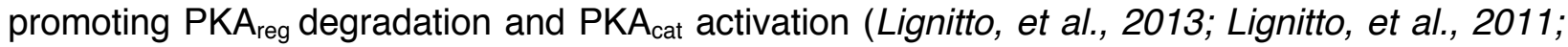
were identified as hits in CRISPR knockout screens for Hh pathway regulators (Breslow, et al., 402 2018; Pusapati, et al., 2018), suggesting that they participate in an ARHGAP36-specific pathway 403 for Gli activation. Determining how these factors contribute to ARHGAP36 action could uncover novel aspects of Gli regulation.

Taken together, our findings provide new insights into the mechanisms that underlie non406 canonical Gli activation by ARHGAP36, and they provide a general framework for understanding 407 ARHGAP36 function. In particular, our studies reveal how ARHGAP36 can translate multiple 408 cellular inputs into distinct signaling outputs. By controlling the expression of ARHGAP36 isoforms 409 and/or ARHGAP36-interacting proteins, cells can direct this signaling protein to specific PKA $\mathrm{A}_{\text {cat }}$ 410 populations and elicit tissue-specific responses. We anticipate that these mechanisms not only 411 contribute to Gli-dependent spinal cord development and medulloblastoma progression but also 412 other $\mathrm{PKA}_{\text {cat }}$-dependent processes in normal physiology and disease. Moreover, the experimental 413 methods utilized for our study could be redeployed to elucidate these unique ARHGAP36 414 functions. 


\section{METHODS}

417 Reagents and cell lines

Antibody sources and working dilutions are listed in Supplementary File 3. Forskolin was and HEK-293T cells were purchased from the American Type Culture Collection.

Expression vectors

The pDONR223 vector was provided by J. Hartley and D. Esposito. The following constructs have been described previously (Rack, et al., 2014; Wright, et al., 2011): Gateway cloning destination vectors pBMN-3xFLAG-IRES-mCherry-DEST, pBMN-mCherry-DEST, and pG-LAP7-DEST, Gateway cloning entry vectors pDONR223-ARHGAP36 (isoforms 1 - 5), and expression vectors pcDNA3.2-ARHGAP36 (isoform 2)-V5, pBMN-3xFLAG-IRES-mCherry, and pBMN-mCherry. pCL-ECO retrovirus packaging vector was purchased from Imegenex. by transferring cDNAs from the appropriate pDONR223 entry vectors into pBMN-3xFLAG-IRESmCherry-DEST in an LR Clonase II (Invitrogen, Waltham, MA)-mediated recombination reaction. Retroviral expression vectors for mCherry-tagged ARHGAP36 isoforms 1 and 2 were produced 434 in an analogous manner with pBMN-mCherry-DEST. Restriction sites were then inserted 435 upstream (Xhol) and downstream (Sacll) of the ARHGAP36 sequence in the initial pBMN436 ARHGAP36 (isoform 1)-mCherry and pBMN-ARHGAP36 (isoform 2)-mCherry products. This was 437 achieved by first amplifying ARHGAP36 cDNA from the pBMN-derived vectors (Supplementary 438 File 3; primers 1 - 3) and then inserting the resulting PCR product into BamHI-digested pBMN439 mCherry using Gibson assembly (New England Biolabs, Ipswich, MA). The resulting pBMN- 
440

441

442

443

444

445

446

447

448

449

450

451

452

453

ARHGAP36-mCherry vectors with Xhol and Sacll restriction sites were subsequently used in all experiments described herein.

Retroviral expression vectors for ARHGAP36 isoform 2 truncation mutants were generated by amplifying the cDNA for each variant from pcDNA3.2-ARHGAP36 (isoform 2)-V5, using primers containing attB adapter sequences (Supplementary File 3; primers 4-8). The PCR products were transferred into pDONR223 in a BP Clonase II (Invitrogen)-mediated recombination reaction, and the ARHGAP36-derived cDNAs in these pDONR223 entry vectors were then transferred to pBMN-3xFLAG-IRES-mCherry-DEST using LR Clonase II. The resulting constructs were also used as templates to amplify cDNAs for the analogous FLAG-tagged ARHGAP36 isoform 3 truncation mutants and the downstream IRES-mCherry sequence (Supplementary File 3; primers 9 and 10). These PCR products were then inserted into Xcmldigested pBMN-ARHGAP36 (isoform 2)-3xFLAG-IRES-mCherry using Gibson assembly.

Individual ARHGAP36 isoform 2 point mutants, with the exception of L171P, were generated using site-directed mutagenesis with PfuUltra II Fusion polymerase (Agilent, Santa Clara, CA) (Supplementary File 3; primers 11-42) and either pBMN-ARHGAP36 (isoform 2)3xFLAG-IRES-mCherry or the pBMN-ARHGAP36 (isoform 2)-mCherry as template. ARHGAP36 isoform 2 L171P mutant constructs were generated by Gibson assembly using a Xhol- and Saclldigested pBMN-ARHGAP36 (isoform 2)-mCherry plasmid, inserts amplified from pBMNARHGAP36 (isoform 2)-mCherry (Supplementary File 3; primers 3 and 43-45), and a doublestranded oligonucleotide encoding the L171P mutation (Integrated DNA Technologies, Coralville, IA) (Supplementary File 3; entry 46) .

Retroviral expression vectors for mCherry-tagged wild-type, C107Y, and L212P ARHGAP36 isoform 3 were generated by amplifying the cDNAs encoding this isoform from the corresponding mutant pBMN-ARHGAP36 (isoform 2)-mCherry plasmids (Supplementary File 3; primers 3 and 47) and amplifying the mCherry tag from pBMN-ARHGAP36 (isoform2)-mCherry 
plasmid (Supplementary File 3; primers 10 and 48). The resulting amplicons were inserted into Xcml-digested pBMN-ARHGAP36 (isoform 2)-3xFLAG-IRES-mCherry using Gibson assembly. Retroviral expression vectors for mCherry-tagged wild-type, C212Y, and L317P ARHGAP36 N2GAP were generated by amplifying the cDNAs encoding ARHGAP36 N2-GAP from the corresponding mutant pBMN-ARHGAP36 (isoform 2)-mCherry plasmids (Supplementary File 3;

470 primers 45 and 49) and inserting into Xhol- and Sacll-digested pBMN-ARHGAP36 (isoform 2)471 mCherry using Gibson assembly.

To generate retroviral expression vectors for LAP-tagged ARHGAP36 constructs, ARHGAP36 cDNA in the pDONR223-ARHGAP36 (isoform 2) entry vector was transferred to pG-

474 LAP7-DEST using LR Clonase II. The cDNA encoding LAP-tagged ARHGAP36 was amplified 475 from the resulting pG-ARHGAP36 (isoform 2)-LAP7 plasmid (Supplementary File 3; primers 10 476 and 50) and inserted into Xcml-digested pBMN-ARHGAP36 (isoform 2)-3xFLAG-IRES-mCherry 477 using Gibson assembly. LAP-tagged L317P ARHGAP36 isoform 2 retroviral expression 478 constructs were generated using an analogous Gibson assembly with ARHGAP36 cDNA 479 amplified from pBMN-ARHGAP36 (isoform 2 L317P)-3xFLAG-IRES-mCherry (Supplementary 480 File 3; primers 50 and 51) and LAP tag cDNA amplified from pG-ARHGAP36 (isoform 2)-LAP7 481 (Supplementary File 3; primers 10 and 48).

With the exception of the constructs generated by site-directed mutagenesis described 483 above, all PCR products were generated with Phusion polymerase (New England Biolabs). All 484 plasmids were sequence-verified.

\section{Retrovirus production}

HEK-293T cells were seeded into individual wells of a 6-well plate at a density of $4881.0 \times 10^{6}$ cells/well. The cells were cultured for 24 hours in HEK-293T growth medium (DMEM 489 containing $10 \%$ fetal bovine serum, $2 \mathrm{mM} \mathrm{L}$-glutamine, $1 \mathrm{mM}$ sodium pyruvate, $100 \mathrm{U} / \mathrm{mL}$ 
penicillin, and $0.1 \mathrm{mg} / \mathrm{mL}$ streptomycin) and then transfected as follows. pBMN vectors containing

491 the appropriate ARHGAP36 construct $(1.33 \mu \mathrm{g})$ and the $\mathrm{pCL}-\mathrm{ECO}$ retrovirus packaging vector $492(0.67 \mu \mathrm{g})$ were diluted in OMEM medium $(75 \mu \mathrm{L})$, and the solution was added to OMEM $(75 \mu \mathrm{L})$ containing $6 \mu \mathrm{L}$ Fugene HD reagent (Promega, Madison, WI). The mixture was incubated at room temperature for 10 minutes and gently added to the growth medium on the cultured cells. After 24 hours, the medium was replaced with DMEM containing $1.8 \mathrm{mM} \mathrm{L-glutamine,} 4 \%$ fetal bovine serum, $6 \%$ calf serum, $1 \mathrm{mM}$ sodium pyruvate, $100 \mathrm{U} / \mathrm{mL}$ penicillin, and $0.1 \mathrm{mg} / \mathrm{mL}$ streptomycin.

Retrovirus-containing supernatant was then collected two times at 20-hour intervals, passed

through a $0.45-\mu \mathrm{m}$ filter, and stored at $-80^{\circ} \mathrm{C}$. Large-scale retrovirus production was conducted using HEK-293T cells seeded on 10-cm plates at a density of $5.0 \times 10^{6}$ cells/well transfected with $6.48 \mu \mathrm{g}$ of the ARHGAP36 construct, $4 \mu \mathrm{g}$ of $\mathrm{pCL}-\mathrm{ECO}$, and $35 \mu \mathrm{L}$ of Fugene HD in $750 \mu \mathrm{L}$ OMEM medium.

502

Generation of ARHGAP36-expressing NIH-3T3 cell lines

$\mathrm{NIH}-3 \mathrm{~T} 3$ cells were seeded into individual wells of 24 -well or 6 -well plates at a density of growth medium (DMEM containing $10 \%$ calf serum, $1 \mathrm{mM}$ sodium pyruvate, $100 \mathrm{U} / \mathrm{mL}$ penicillin, and $0.1 \mathrm{mg} / \mathrm{mL}$ streptomycin) and then transduced with $4 \mu \mathrm{g} / \mathrm{mL}$ polybrene and retrovirus for the 508 appropriate ARHGAP36-3xFLAG-IRES-mCherry construct to achieve a multiplicity of infection $509(\mathrm{MOI})<0.5$. After 24 hours, the medium was exchanged, and the cells were expanded for fluorescence-activated cell sorting (FACS).

For FACS, the cells were washed with PBS buffer, dissociated with TrypLE (Invitrogen)

512 for $3-5$ minutes at $37^{\circ} \mathrm{C}$, and centrifuged at $106 \mathrm{~g}$ for 5 minutes at $4{ }^{\circ} \mathrm{C}$. Cell pellets were then 513 resuspended in FACS buffer (PBS containing 1\% calf serum), passed through a $70-\mu m$ cell 514 strainer (BD Biosciences, San Jose, CA), and added to round-bottom FACS tubes. Cell 
515 populations with comparable mCherry fluorescence intensities were then obtained using a BD

516 FACSAria II (532-nm laser and 600-nm longpass filter, or 561-nm laser and 610/20-nm bandpass

517 filter) or BD Influx (561-nm laser and 610/20-nm bandpass filter) sorter.

518

Quantitative reverse transcription-PCR (qRT-PCR) analyses

$\mathrm{NIH}-3 \mathrm{~T} 3$ cell lines expressing the indicated FLAG-tagged ARHGAP36 constructs were seeded into 6-well plates at a density of $5.2 \times 10^{5}$ cells/well and cultured in NIH-3T3 growth confluent cells were treated with NIH-3T3 low-serum medium (DMEM containing $0.5 \%$ calf serum, sodium pyruvate and antibiotics) with or without $10 \% \mathrm{SHH}-\mathrm{N}$-conditioned medium for 30 hours.

525 The media was replaced with ice-cold PBS, and the cells were collected by manual scraping. blot analyses) and centrifuged at $750 \mathrm{~g}$ for 7 minutes at $4{ }^{\circ} \mathrm{C}$.

Cell pellets were prepared for qRT-PCR analyses as follows. RNA was isolated using the Monarch Total RNA miniprep kit (New England BioLabs), and equivalent amounts of RNA were used to synthesize cDNA using the SuperScript III First-Strand Synthesis System (Invitrogen).

TaqMan probes: Gli1-Mm00494645_m1, Beta-2-Microglobulin-Mm00437762_m1 (Applied

533 Biosystems, Waltham, MA). Gene expression levels were normalized to $\beta$-2-microglobulin. For 534 ARHGAP36 isoform and truncation mutant analyses, the normalized gene expression levels were compared to that of uninfected cells, and for point mutant analyses, compared to that of wild-type ARHGAP36. The resulting gene expression levels were averaged across three biological

537 replicates, and $P$ values were determined using either a Student's one-tailed t-test (ARHGAP36 isoform and truncation mutant analyses) or two-tailed t-test (point mutant analyses). 


\section{Western blot analyses}

Cell pellets were resuspended in 1x Laemmli sample buffer (10\% glycerol, $2 \%$ SDS, $17 \mathrm{mM}$ DTT, 0.01\% bromophenol blue, $60 \mathrm{mM}$ Tris- $\mathrm{HCl}, \mathrm{pH} 6.8$, and protease and phosphatase inhibitors (Roche)). After incubation for 20 minutes at $4^{\circ} \mathrm{C}$, cell lysates were boiled for 10 minutes and sonicated in a water bath for 15 seconds. Equivalent amounts of total protein per lysate were loaded onto Criterion XT 4-12\% Bis-Tris polyacrylamide gels (Bio-Rad, Hercules, CA), transferred onto PVDF membranes (Bio-Rad), and detected using the antibodies listed in Supplementary File 3 with either SuperSignal West Dura or SuperSignal Femto kits (Pierce, Waltham, MA) and a ChemiDoc XRS imaging system (Bio-Rad). Band intensities were quantified using ImageLab software (Bio-Rad) and normalized to KPNB1 band intensities in the corresponding sample. For each replicate, the normalized band intensity in each condition was normalized to that of uninfected cells. The resulting relative band intensities for each condition was averaged across three biological replicates, and $P$ values were determined using a Student's two-tailed t-test.

\section{Immunofluorescence studies}

The subcellular localizations of FLAG-tagged ARHGAP36 constructs were assessed as follows. NIH-3T3 cells were seeded onto individual wells of a 6-well plate at a density of $2.0 \times 10^{5}$ cells/well. Cells were cultured for 24 hours in NIH-3T3 growth medium, then transduced with $4 \mu \mathrm{g} / \mathrm{mL}$ polybrene and retrovirus for the appropriate ARHGAP36-3xFLAG-IRES-mCherry construct to achieve an $\mathrm{MOI}<0.5$. After 24 hours, cells were re-seeded at a 1:8 dilution into 24well plates containing poly-D-lysine-coated 12-mm glass coverslips and cultured for 1-2 days in growth medium. Cells were fixed in PBS containing 4\% paraformaldehyde for 10 minutes at room temperature and washed 3 times with PBS. Cells were next permeabilized with PBS containing 0.5\% Triton X-100 for 5 minutes, washed 2 times with PBS, and incubated in blocking buffer (PBS containing $1 \% \mathrm{BSA}$ and $0.1 \%$ Triton $\mathrm{X}-100$ ) for 1 hour at room temperature. The cells were then 
incubated for 1 hour at room temperature with primary antibodies diluted in blocking buffer, washed $4 \times 5$ minutes with PBS containing $0.1 \%$ Triton X-100, incubated for 1 hour with the appropriate secondary antibodies diluted in PBS containing $0.2 \%$ Triton X-100, and washed $4 \times$ Prolong Gold Antifade reagent with DAPI (Invitrogen).

The subcellular localizations of mCherry-tagged ARHGAP36 isoform 3 and N2-GAP constructs were assessed as follows. SHH-EGFP cells were seeded into individual wells of a 24well plate at a density of $7.5 \times 10^{4}$ cells/well. The cells were cultured for 24 hours in SHH-EGFP growth medium (NIH-3T3 growth medium containing $150 \mu \mathrm{g} / \mathrm{mL}$ zeocin) for 24 hours and then

574 transduced with $4 \mu \mathrm{g} / \mathrm{mL}$ polybrene and retrovirus for the appropriate ARHGAP36-mCherry 575 construct to achieve an $\mathrm{MOI}<0.5$. After 24 hours, the cells were passaged into a new 24 -well plate at a 1:1.5 dilution and cultured in growth medium for an additional 2 days to achieve $100 \%$ confluency. Confluent cells were then treated for 24 hours with SHH-EGFP low-serum medium

578 (DMEM containing $0.5 \%$ calf serum, sodium pyruvate, zeocin, and antibiotics). Cells were next 579 passaged at a 1:3 dilution into 24-well plates containing poly-D-lysine-coated 12-mm glass 580 coverslips and cultured for 1 day in growth medium. Cells were then fixed, blocked, 581 immunostained, and mounted as described above. The subcellular localizations of mCherry582 tagged isoform 2 constructs were similarly assessed, with the exception that the cells were 583 passaged onto coverslip-containing 24-well plates 24 hours after retroviral transduction then cultured for two days prior to being fixed.

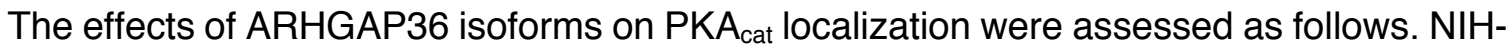
3T3 cell lines stably expressing FLAG-tagged ARHGAP36 isoforms 2 or 3 were seeded into 24587 well plates containing poly-D-lysine-coated $12-\mathrm{mm}$ glass coverslips at a density of $1.2 \times 10^{5}$ 588 cells/well. Cells were then cultured in NIH-3T3 growth medium for 2 days, at which time the cells were fixed in PBS containing $2 \%$ paraformaldehyde for 20 minutes at room temperature and then 
590 treated with methanol for 5 minutes at $-20^{\circ} \mathrm{C}$. The fixed cells were incubated in blocking buffer

591 for 1 hour at room temperature and incubated with primary antibodies diluted in blocking buffer

592 overnight at $4{ }^{\circ} \mathrm{C}$. Subsequent PBS washes, secondary antibody incubation, and mounting were 593 conducted as described above.

$594 \quad$ Fluorescence images were obtained using either a Zeiss LSM 700 or 800 confocal 595 microscope equipped with a 63x oil-immersion objective. Maximum-intensity Z-stack projections 596 were created using either ZEN Black (Zeiss, Oberkochen, Germany), ZEN Blue (Zeiss), or FIJI

597 (Schindelin, et al., 2012) software, fluorescence intensities were adjusted using FIJl, and images were cropped using Photoshop CC (Adobe, San Jose, CA).

Luciferase assays

SHH-LIGHT2 cells were seeded into $10-\mathrm{cm}$ plates at a density of $1.0 \times 10^{6}$ cells/plate and and $500 \mu \mathrm{g} / \mathrm{mL}$ G418). After 24 hours of growth, cells were transduced with $4 \mu \mathrm{g} / \mathrm{mL}$ polybrene 604 and retrovirus for either the appropriate ARHGAP36-3xFLAG-IRES-mCherry construct or for a 3xFLAG-IRES-mCherry construct for another 24 hours. The cells were then re-seeded into individual wells of a 96-well plate at a density of $3.5 \times 10^{4}$ cells/well. All cells were cultured for an additional 24 hours, at which time the fully confluent cells were treated for 30 hours with varying 608 conditions of forskolin in SHH-LIGHT2 low-serum medium (DMEM containing 0.5\% calf serum, $6091 \%$ sodium pyruvate, zeocin, G418, and antibiotics) with or without $10 \%$ SHH-N-conditioned medium. The cells were then lysed and luciferase activities were measured using a Dual-

611 Luciferase Reporter Assay System (Promega) on a Veritas luminometer (Turner BioSystems, 612 Sunnyvale, CA). At least three biological replicates were conducted for each condition. 
Mutant library generation

616

A library encoding ARHGAP36 isoform 2 mutants was created via error-prone PCR (epPCR) using the GeneMorph II Random Mutagenesis Kit (Agilent). To determine the optimal epPCR conditions for library generation, the mutation frequency was estimated for epPCRs consisting of $15,20,25$, or 29 cycles. All reactions were conducted according to the manufacturer's instructions, using $1.64 \mu \mathrm{g}$ of the pBMN-ARHGAP36 (isoform2)-mCherry plasmid as template, $0.4 \mu \mathrm{M}$ of each primer (Supplementary File 3; primers 45 and 52), and 4\% DMSO. The product yield for each condition was estimated by resolving $10 \%$ of the reaction on a $1 \%$ 623 EtBr-agarose gel and quantifying the band intensity of the resulting amplicon with ImageLab 624 software (Bio-Rad). The 1.6-kb amplicon was gel-extracted using the QiaQuick Gel Extraction Kit 625 (Qiagen, Hilden, Germany) and ligated into a Xhol- and Sacll-digested pBMN-ARHGAP36 (isoform 2)-mCherry vector using Gibson assembly. To estimate the mutation frequency for each epPCR-generated library, XL-10 Gold E. coli (Agilent) were chemically transformed with 1:4 diluted Gibson assembly products and plated onto ampicillin-agarose plates. Forty colonies from each plate were sequenced using rolling circle amplification and Sanger sequencing (Sequetech, Mountain View, CA) (Supplementary File 3; primers 53-58). High-quality ARHGAP36 reads were aligned to the coding sequence for wild-type ARHAGP36 isoform 2, and the number of nucleotide mutations within the coding sequence was counted for each read. This analysis yielded the distribution of mutated nucleotides across the library. scale library using the 15-cycle epPCR and Gibson assembly strategy described above. The

637 undiluted Gibson reaction $(8 \mu \mathrm{L})$ was electroporated into $160 \mu \mathrm{L}$ of MegaX $\mathrm{DH} 10 \mathrm{~B} \mathrm{~T}^{\mathrm{R}}$ 638 Electrocomp cells (Invitrogen). The electroporated cells were immediately transferred to $480 \mathrm{~mL}$ 639 of Superior Broth containing $75 \mu \mathrm{g} / \mathrm{mL}$ ampicillin, and $100 \mu \mathrm{L}$ of the culture was plated on 
640 ampicillin-agar plates to estimate the number of colony-forming units. The final library was found

641 to contain approximately $4 \times 10^{5}$ colony-forming units, which corresponds to an equivalent number

642 of library elements. The liquid culture was incubated at $30^{\circ} \mathrm{C}$ until it reached an $\mathrm{OD}_{600}$ of 1 , after

643 which plasmids were isolated using the NucleoBond Xtra Midi Plus Kit (Macherey-Nagel, Düren, 644 Germany).

645 Retroviral medium harboring the ARHGAP36 mutant library was generated in the following 646 manner. One $10-\mathrm{cm}$ plate of HEK-293T cells at $90 \%$ confluency was transfected with $6.5 \mu \mathrm{g}$ of 647 the pBMN-ARHGAP36 (isoform 2)-mCherry mutant library and $4 \mu \mathrm{gg}$ of pCL-ECO using the 648 FuGene HD transfection reagent (Promega). The medium was replaced after 24 hours with 649 DMEM containing $1.8 \mathrm{mM}$ L-glutamine, 4\% fetal bovine serum, $6 \%$ calf serum, $1 \%$ sodium 650 pyruvate, $100 \mathrm{U} / \mathrm{mL}$ penicillin, and $0.1 \mathrm{mg} / \mathrm{mL}$ streptomycin. Retrovirus-containing supernatant was then collected two times at 24-hour intervals, passed through a $0.45-\mu \mathrm{m}$ filter, and stored at $652-80^{\circ} \mathrm{C}$.

FACS-based screening

SHH-EGFP cells were seeded onto a $15-\mathrm{cm}$ plate at a density of $1.0 \times 10^{6}$ cells/plate and cultured in SHH-EGFP growth medium for 2 days, then transduced with the retroviral library of ARHGAP36 mutants and $4 \mu \mathrm{g} / \mathrm{mL}$ polybrene to achieve an $\mathrm{MOI}<0.5$. After 24 hours, the cells were expanded to $4 \times 15-\mathrm{cm}$ plates and cultured for an additional 2 days. SHH-EGFP cells treated with $10 \% \mathrm{SHH}-\mathrm{N}$-conditioned media or transduced with retrovirus encoding wild-type ARHGAP36 isoform 2 served as positive controls for flow cytometry. For negative controls, untreated SHH-

661 EGFP cells or cells transduced with retrovirus encoding ARHGAP36 isoform 1 were used. To isolate mCherry+ cells by FACS, the transduced SHH-EGFP cells were washed with 663 PBS, dissociated with TrypLE for 3-5 minutes at $37^{\circ} \mathrm{C}$, and centrifuged at $750 \mathrm{~g}$ for 7 minutes at 
passed through a $70-\mu \mathrm{m}$ cell strainer, and added to round-bottom FACS tubes. Cell sorting was

666 performed on a BD FACSAria II configured with a 561-nm laser, a 595-nm longpass filter, and a

667 616/23-nm bandpass filter for mCherry detection. Data was collected with FACSDiva software

668 (BD Biosciences) and analyzed using FlowJo (FlowJo, Ashland, Oregon). $2.7 \times 10^{7}$ cells were 669 analyzed by FACS analysis.

This first sort produced a population of $3 \times 10^{6}$ mCherry+ cells, which were cultured for

6713 days until they reached $100 \%$ confluency. The cells were then cultured in SHH-EGFP low-

672 serum medium to enable ARHGAP36-mediated Gli activation. After 24 hours, cells were

673 expanded at a 1:2 dilution, cultured for 24 hours to achieve full confluency, and serum-starved

674 again for another 24 hours.

675 To isolate cells expressing inactive forms of ARHGAP36 isoform 2 (mCherry+/EGFP-), $1.1 \times 10^{7}$ cells from the expanded mCherry+ population were washed and dissociated as described above. Approximately $3 \times 10^{6}$ cells were separated and expanded to $4 \times 10^{7}$ cells to establish a pre-selection population, which was then washed with PBS, dissociated, and pelleted by centrifugation at $750 \mathrm{~g}$ for 7 minutes at $4{ }^{\circ} \mathrm{C}$. The pellet was stored at $-80{ }^{\circ} \mathrm{C}$ until used for genomic DNA extraction. The remaining $8 \times 10^{6}$ mCherry+ cells were analyzed by FACS to select for those expressing inactive ARHGAP36-mCherry mutants. Cells were sorted as described above using the BD FACSAria II configured with a 488-nm dye laser, a 495-nm longpass filter, and a 530/30-nm bandpass filter for EGFP detection and the laser/filter configurations described above for mCherry detection. Approximately $4 \times 10^{5}$ mCherry+/EGFP-cells were obtained from this second sort, and they were cultured for 2 days to reach full confluency and then subjected to 2 rounds of serum starvation. FACS sorting of this enriched population yielded $1.6 \times 10^{5}$ 687 mCherry+/EGFP- cells, which were expanded, frozen in SHH-EGFP growth medium containing $68810 \%$ DMSO, and stored in liquid nitrogen. 
We then assessed if the mCherry+/EGFP- cells were still capable of Gli-dependent EGFP

690

691

692

693

694

695

696

697

698

699

700

701

702

703

704

705

706

707

708

709

710

711

712

713

expression under canonical Hh pathway activation conditions. Frozen aliquots of cells from the third sort were thawed, expanded, and subjected to two rounds of serum starvation. Approximately $1.0 \times 10^{7}$ cells were sorted with a BD FACSAria llu configured with a 488-nm laser, a 502-nm longpass filter, and a 525/50-nm bandpass filter for EGFP detection and a 488-nm laser, a 595-nm longpass filter, and a 610/20-nm bandpass filter for mCherry detection. $1.0 \times 10^{6}$ mCherry+/EGFP- cells were collected and cultured for 7 days to achieve full confluency. The cells were then treated with 200 nM SAG in SHH-EGFP low-serum medium for 24 hours, expanded at a 1:2 dilution, cultured for 24 hours to enable full confluency, and treated again with 200 nM SAG for 24 hours. $2 \times 10^{7}$ cells were then sorted with the BD FACSAria llu to obtain $3 \times$

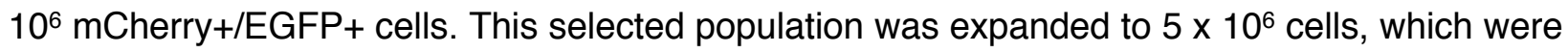
then washed with PBS, dissociated, and pelleted by centrifugation at $750 \mathrm{~g}$ for 7 minutes at $4{ }^{\circ} \mathrm{C}$. The pellet was stored at $-80^{\circ} \mathrm{C}$ until used for genomic DNA extraction.

\section{Deep-sequencing analyses of pre- and post-selection pools}

Genomic DNA was extracted from frozen pellets using the QIAamp DNA Blood Maxi Kit (Qiagen) according to manufacturer's instructions. For each sample, ARHGAP36 inserts were isolated from genomic DNA by PCR using $500 \mathrm{ng}$ of genomic DNA, $0.5 \mu \mathrm{M}$ each of primer (Tables S3, primers 59 and 60), $0.2 \mu \mathrm{M}$ dNTP mix, and Phusion High-Fidelity DNA Polymerase in HF buffer (New England BioLabs). A total of 182 PCRs were used to isolate ARHGAP36 inserts from $91 \mu \mathrm{g}$ of pre-selection genomic DNA, while 89 PCRs were used to isolate inserts from $45 \mu \mathrm{g}$ of post-selection genomic DNA. For each condition, the respective reactions were pooled, purified using the QIAquick PCR Purification Kit (Qiagen), and resolved on a $0.8 \% \mathrm{EtBr}$-agarose gel. The 1.6-kb amplicon was then gel-extracted using the QIAquick Gel Extraction Kit. Amplicons were quantified using a Bioanalyzer 2100 with high-sensitivity DNA kits (Agilent), sheared into 150-bp 
714 fragments with an S220 focused-ultrasonicator (Covaris, Woburn, MA), and sequenced on a

715 NextSeq 500 Sequencer using High-Output v2 kits (Illumina, San Diego, CA). Raw data have

716 been deposited into the Dryad Digital Repository with the dataset identifier

717 10.5061/dryad.dz08kprv9.

FASTQ files were aligned to the wild-type ARHGAP36 coding sequence (Bowtie2 v2.3

719 (Langmead, et al., 2012) and sorted by read name (SAMtools v1.3.1) (Li, Heng, et al., 2009). The

720 resulting mapped and sorted reads were then analyzed with an in-house Python script

721 (Supplementary File 4). Briefly, high-quality reads were aligned to the wild-type ARHGAP36

722 coding sequence. Reads with greater than 3 high-quality mutations or with internal stop codons

723 were discarded from further analysis. The remaining reads were translated, identifying the

724 ARHGAP36 amino acid mutations present in the population. For each residue, the fold-change in

725 its mutation frequency between the pre- and post-selection populations was calculated.

726

Flow cytometry-based assays

SHH-EGFP cells were seeded into individual wells of a 24-well plate at a density of

$7.5 \times 10^{4}$ cells/well and cultured for 24 hours in SHH-EGFP growth medium. The cells were then

transduced with retrovirus for the appropriate ARHGAP36-mCherry construct and $4 \mu \mathrm{g} / \mathrm{mL}$

polybrene to achieve an $\mathrm{MOI}<0.5$. An uninfected condition was also prepared as a negative

control. After 24 hours, the cells were passaged onto a new 24-well well at a 1:1.5 dilution and

733 cultured in growth medium for an additional 2 days to achieve $100 \%$ confluency. Confluent cells

734 were then treated with SHH-EGFP low-serum medium for 24 hours. Cells were then passaged at

735 a 1:1.5 dilution onto a new 24-well well and cultured for 24 hours to achieve full confluency for a

736 second round of 24-hour serum-starvation with or without $\mathrm{SHH}-\mathrm{N}$-conditioned medium.

737 For flow cytometry analyses, the cells were washed with PBS, dissociated with TrypLE for

$738 \quad 3-5$ minutes at $37^{\circ} \mathrm{C}$, and centrifuged $750 \mathrm{~g}$ for 7 minutes at $4^{\circ} \mathrm{C}$. Cell pellets were resuspended 
739

740

741

742

743

744

745

746

747

748

749

750

751

752

753

754

755

756

757

758

759

760

761

762

763

in FACS buffer (PBS containing 1\% calf serum) and analyzed on a DxP FACScan (561-nm laser and 616/25-nm bandpass filter for mCherry detection; 488-nm laser, 560-nm shortpass filter, and 525/50-nm bandpass filter for EGFP detection) or a BD LSRII (561-nm laser, 600-nm longpass band filter, and a 610/20-nm bandpass filter for mCherry detection; 488-nm laser, 505-nm longpass band filter, and 525/50-nm bandpass filter for EGFP detection). Data was collected with Cypod (Cytek, Fremont, CA) and FACSDiva software and analyzed using FlowJo. Fluorescence data was collected for at least $2.5 \times 10^{4}$ cells, and three biological replicates were analyzed for each condition.

Data analyses for all conditions except for the uninfected controls excluded mCherrycells, which is indicative of a lack of ARHGAP36 expression, and the median EGFP fluorescence was calculated to measure Gli activity in each condition. For each replicate, the median EGFP fluorescence was normalized to that of wild-type ARHGAP36-expressing cells, and a Student's one-tailed t-test was used to identify mutations that significantly altered median EGFP fluorescence levels $(P \leq 0.05)$.

\section{Tandem affinity purification and quantitative proteomics}

$\mathrm{NIH}-3 \mathrm{~T} 3$ cells were seeded onto $15-\mathrm{cm}$ plates ( 6 per condition) at a density of $2 \times 10^{6}$ cells/plate and cultured in NIH-3T3 growth medium. After 24 hours, cells were transduced with retrovirus for either wild-type or L317P ARHGAP36 with a C-terminal LAP tag and $4 \mu \mathrm{g} / \mathrm{mL}$ polybrene to achieve an $\mathrm{MOI}>1.5$. The media was exchanged for growth medium after 4 hours, and cells were cultured for an additional 20 hours. Cells were then washed with cold PBS, manually scraped off each dish, and transferred into Falcon tubes. Cell suspensions expressing the same ARHGAP36 construct were combined, and $0.5 \%$ of the resulting pool was reserved for downstream flow cytometry analyses to confirm the $\mathrm{MOI}$. The remaining cells were centrifuged at $750 \mathrm{~g}$ for 7 minutes at $4{ }^{\circ} \mathrm{C}$. The supernatant was aspirated, and the remaining cell pellet was 
764

765

766

767

768

769

770

771

772

773

774

775

776

777

778

779

780

781

782

783

784

785

786

787

788

flash frozen in liquid nitrogen and stored at $-80{ }^{\circ} \mathrm{C}$ prior to LAP-tagged mediated tandem affinity purification. Three biological replicates were conducted for each wild-type and L317P ARHGAP36-LAP comparison.

Tandem affinity purifications and mass spectrometry analyses were conducted as described previously (Ding, et al., 2016; Hsu, et al., 2019; Kanie, et al., 2017; Li, Bin, et al., 2017; Torres, et al., 2009; Wright, et al., 2011). Pellets of ARHGAP36-LAP-expressing cells were resuspended in LAP resuspension buffer (300 mM KCl, 50 mM HEPES-KOH (pH 7.4), 1 mM EGTA, $1 \mathrm{mM} \mathrm{MgCl}_{2}, 10 \%$ glycerol, $0.5 \mathrm{mM}$ DTT, and protease inhibitors (Thermo Scientific)). Cells were lysed with the gradual addition of $10 \%$ NP-40 to a final concentration of $0.3 \%$, followed by a 10 minute incubation at $4{ }^{\circ} \mathrm{C}$. The lysate was then centrifuged at $27,000 \mathrm{~g}$ at $4{ }^{\circ} \mathrm{C}$ for 10 minutes, and the resulting supernatant was centrifuged at $100,000 \mathrm{~g}$ for 1 hour at $4{ }^{\circ} \mathrm{C}$. The high-speed supernatant was next incubated with anti-GFP-antibody-coupled beads (Ding, et al., 2016; Hsu, et al., 2019; Kanie, et al., 2017; Li, Bin, et al., 2017; Torres, et al., 2009; Wright, et al., 2011) for 1 hour at $4{ }^{\circ} \mathrm{C}$ to capture GFP-tagged proteins. The beads were washed five times with LAP200N buffer (200 mM KCl, 50 mM HEPES-KOH (pH 7.4), 1 mM EGTA, 1 mM MgCl $2,10 \%$ glycerol, protease inhibitors, and $0.05 \%$ NP40) and incubated with PreScission protease in LAP200N buffer at $4{ }^{\circ} \mathrm{C}$ for 16 hours. All subsequent steps were performed in a laminar flow hood. PreScission protease-eluted supernatant was added to S-protein agarose beads (EMD Millipore, Burlington, MA) and incubated rocking for 3 hours at $4{ }^{\circ} \mathrm{C}$. S-protein agarose beads were then washed three times with LAP200N buffer and twice with LAP100 buffer (100 mM KCl, $50 \mathrm{mM}$ HEPES-KOH (pH 7.4), 1 mM EGTA and 10\% glycerol). Beads were stored in 50mM HEPES (pH 7.5), $1 \mathrm{mM}$ EGTA, $1 \mathrm{mM} \mathrm{MgCl} 2,10 \%$ glycerol at $4{ }^{\circ} \mathrm{C}$ prior to on-bead digestion.

Proteins were eluted from S-protein agarose beads with an on-bead reduction, alkylation, and tryptic digestion as follows. Samples were reduced with $10 \mathrm{mM}$ DTT in ammonium bicarbonate for an initial 5-minute incubation at $55^{\circ} \mathrm{C}$ followed by 25 minutes at room temperature. 
789 The proteins were then alkylated with a 30 -minute incubation in $30 \mathrm{mM}$ acrylamide at room

790 temperature, and finally eluted from the beads with an overnight digest performed at room 791 temperature using Trypsin/LysC (Promega) and 0.02\% ProteaseMax (Promega). The digests 792 were acidified with $1 \%$ formic acid, de-salted with C18 Monospin reversed phase columns (GL 793 Sciences, Tokyo, Japan), dried on a SpeedVac, and reconstituted in $12.5 \mu \mathrm{L}$ of $2 \%$ acetonitrile 794 and $0.1 \%$ formic acid. $4 \mu \mathrm{L}$ of each sample were used for liquid-chromatography-mass 795 spectrometry analyses performed on an Acquity M-Class UPLC (Waters Corporation, Milford, MA) and either an Orbitrap Q-Exactive HFX mass spectrometer (Thermo Scientific, San Jose, CA) or an Orbitrap Fusion Tribrid mass spectrometer (Thermo Scientific). For each biological replicate,

798 the sample from the ARHGAP36 L317P-expressing cells was run immediately before that of the 799 wild-type ARHGAP36-expressing cells. Analysis of the resulting .RAW data files was conducted using Byonic (Protein Metrics, San Carlos, CA), with the assumption of tryptic proteolysis and a maximum allowance of two missed cleavage sites. Precursor and MS/MS fragment mass 802 accuracies were held within $12 \mathrm{ppm}$ and $0.4 \mathrm{Da}$, respectively. A false discovery rate of $1 \%$ was 803 used for protein identification (Elias, et al., 2007). Raw data have been deposited to the 804 ProteomeXchange Consortium via the PRIDE partner repository with the dataset identifier PXD019056 and 10.6019/PXD019056.

The resulting list of identified proteins was compared to an NCBI FASTA database containing all mouse proteomic isoforms with the exception of the tandem affinity bait construct sequence and common contaminant proteins. Post-processing of spectral counts was conducted with an in-house $\mathrm{R}$ script (Supplementary File 5). For each protein, spectral counts detected across all isoforms were combined and normalized to the mean amino acid length of all isoforms.

811 The resulting normalized spectral count was divided by the sum of normalized spectral counts 812 calculated for all proteins in the pulldown sample, generating a normalized spectral abundance 813 factor (NSAF) for each protein. To account for variability in bait ARHGAP36 expression across 
814 different pulldown samples, the NSAF of each protein was divided by that of the bait ARHGAP36

815 (relative NSAF). Proteins that were detected in all biological replicates for a given condition were

816 tabulated, resulting in a dataset of 566 candidate ARHGAP36-binding proteins. For each protein,

817 the fold-change in relative NSAF between the wild-type and L317P mutant ARHGAP36 pulldown

818 samples of the same replicate was calculated. The average fold-change in relative NSAF across

819 of all three replicates was then calculated for each protein.

820

To assess the robustness of our comparative interactome analyses, we calculated a

modified Z score that compares the protein enrichment in either interactome (wild-type vs. L317P

822

ARHGAP36) against the experimental variability in protein abundance measurements. Protein

enrichment was represented by the $\log _{2}$-transformation of the average fold change in relative

NSAF between wild-type and L317P ARHGAP36 interactomes [ $\log _{2}$ (WT NSAF:L317 NSAF)]. We

estimated the error in measuring protein abundance in a given interactome by normalizing the

relative NSAFs for each replicate to the average value across all three replicates (mean-

827

normalized relative NSAFs). This transformation approximates how much the variation between

replicates can contribute to an apparent fold change. To place equal weight on upward and downward variations from the mean, we calculated the absolute value of the $\log _{2}$-transformed mean-normalized relative NSAFs. These calculations were conducted for the relative NSAFs of a protein in both the wild-type and L317P mutant ARHGAP36 interactomes, and the two resulting values were summed to produce a final error estimate. The modified $Z$ score of each protein was calculated by dividing the $\log _{2}$ (WT NSAF:L317 NSAF) by the final error estimate and then

834 calculating the absolute value of the resulting quotient. Proteins with higher modified Z scores are 835 those that are enriched in a given interactome to a degree that is greater than the estimated 836 experimental error. 
bioRxiv preprint doi: https://doi.org/10.1101/2020.05.14.094961; this version posted May 24, 2020. The copyright holder for this preprint (which was not certified by peer review) is the author/funder. All rights reserved. No reuse allowed without permission.

839 Statistical analyses

840 Biological replicates are defined as experimental samples that are capable of biological

841 variance, and technical replicates are defined as those for which experimental variance is solely

842 dependent on measurement accuracy.

843 


\section{4}

845

846

847

848

849

850

851

852

853

854

855

856

857

858

859

860

861

862

863

864

865

866

867

\section{SUPPLEMENTAL DATA}

Figure 4 - figure supplement 1

Figure 6 - figure supplement 1

Supplementary File 1. Mutation frequencies in pre- and post-selection populations

Supplementary File 2. Wild-type and L317P ARHGAP36 isoform 2 interactomes

Supplementary File 3. Antibody and primer resources

Supplementary File 4. Python script for ARHGAP36 mutagenesis screen analysis

Supplementary File 5. R script for ARHGAP36-LAP mass spectrometry analysis

\section{ACKNOWLEDGEMENTS}

This work was supported by the National Institutes of Health (R35 GM127030 to J.K.C.; S10 RR025518-01 and S10 RR027431-01 to the Stanford Shared FACS Facility; and P30 CA124435 to Stanford University Mass Spectrometry), the Rachel Molly Markoff Foundation (J.K.C.), and an Alex's Lemonade Stand Foundation Young Investigator Award (J.N.). Cell sorting and flow cytometry analyses were conducted at the Stanford Shared FACS Facility. Deep sequencing analyses were performed at the Stanford Functional Genomics Facility. Quantitative proteomics were executed at the Vincent Coates Foundation Mass Spectrometry Laboratory, Stanford University Mass Spectrometry.

\section{AUTHOR CONTRIBUTIONS}

P.R. N. designed and conducted experiments, analyzed the data, and wrote the paper.

T.K. developed software to analyze deep sequencing data. N.A.M. performed tandem affinity purifications. J.N. generated expression vectors for ARHGAP36 isoforms and isoform 2 truncation mutants. J.D. contributed to the development of software to analyze proteomic data. P.K.J. 
868 provided resources and supervision for proteomics experiments. J.K.C. designed the 869 experiments, analyzed the data, and wrote the paper.

870

871 COMPETING FINANCIAL INTERESTS

$872 \quad$ The authors declare no competing financial interests.

873 


\section{REFERENCES}

875 Ågren, M., Kogerman, P., Kleman, M.I., Wessling, M. \& Toftgård, R. 2004. Expression of the

876

877

878

879

880

881

882

883

884

885

886

887

888

889

890

891

892

893

894

895

896

897 PTCH1 tumor suppressor gene is regulated by alternative promoters and a single functional Gli-binding site. Gene

330: $\quad 101-114$.

doi: https://doi.org/10.1016/j.gene.2004.01.010.

Amin, E. et al. 2016. Deciphering the molecular and functional basis of RHOGAP family proteins: A systematic approach toward selective inactivation of $\mathrm{RHO}$ family proteins. Journal of Biological Chemistry 291: 20353-20371. doi: https://doi.org/10.1074/jbc.M116.736967.

Bai, C.B., Stephen, D. \& Joyner, A.L. 2004. All mouse ventral spinal cord patterning by Hedgehog is Gli dependent and involves an activator function of Gli3. Developmental Cell 6: 103115. doi: https://doi.org/10.1016/S1534-5807(03)00394-0.

Barzi, M., Berenguer, J., Menendez, A., Alvarez-Rodriguez, R. \& Pons, S. 2009. Sonichedgehog-mediated proliferation requires the localization of PKA to the cilium base. Journal of Cell Science 123: 62-69. doi: https://doi.org/10.1242/jcs.060020, PMID: 5661997.

Beauchamp, E. et al. 2009. GLI1 is a direct transcriptional target of EWS-FLI1 oncoprotein. The Journal of Biological Chemistry 284: 9074-9082. doi: https://doi.org/10.1074/jbc.M806233200, PMID: 19189974.

Beckmann, P.J. et al. 2019. Sleeping beauty insertional mutagenesis reveals important genetic drivers of central nervous system embryonal tumors. Cancer Research 79: 905-917. doi: https://doi.org/10.1158/0008-5472.CAN-18-1261, PMID: 30674530.

Breslow, D.K. et al. 2018. A CRISPR-based screen for Hedgehog signaling provides insights into ciliary function and ciliopathies. Nature Genetics 50: 460-471. doi: https://doi.org/10.1038/s41588-018-0054-7. 
Briscoe, J., Pierani, A., Jessell, T.M. \& Ericson, J. 2000. A homeodomain protein code specifies progenitor cell identity and neuronal fate in the ventral neural tube. Cell 101: 435-445. doi: https://doi.org/10.1016/S0092-8674(00)80853-3, PMID: 10830170.

Buonamici, S. et al. 2010. Interfering with resistance to smoothened antagonists by inhibition of the PI3K pathway in medulloblastoma. Sci. Transl. Med. 10.1126/scitransImed.3001599 51-70. doi: https://doi.org/10.1126/scitransImed.3001599, PMID: 20881279.

Chávez, M. et al. 2015. Modulation of ciliary phosphoinositide content regulates trafficking and Sonic Hedgehog signaling output. Developmental Cell 34: 338-350. doi:

Chen, J.K., Taipale, J., Young, K.E., Maiti, T. \& Beachy, P.A. 2002. Small molecule modulation of Smoothened activity. Proceedings of the National Academy of Sciences of the United States of America 99: 14071-14076. doi: https://doi.org/10.1073/pnas.182542899.

Dai, P. et al. 1999. Sonic hedgehog-induced activation of the Gli1 promoter is mediated by GLI3.

Dennler, S. et al. 2007. Induction of sonic hedgehog mediators by transforming growth factorbeta: Smad3-dependent activation of Gli2 and Gli1 expression in vitro and in vivo. Cancer Research 67: 6981-6986. doi: https://doi.org/10.1158/0008-5472.CAN-07-0491, PMID:

Ding, S. et al. 2016. Comparative proteomics reveals strain-specific $\beta$-TrCP degradation via rotavirus NSP1 hijacking a host Cullin-3-Rbx1 complex. PLoS Pathogens 12: e1005929. doi: https://doi.org/10.1371/journal.ppat.1005929.

920 Dorn, K.V., Hughes, C.E. \& Rohatgi, R. 2012. A Smoothened-Evc2 complex transduces the Hedgehog signal at primary cilia. Developmental Cell 23: 823-835. doi: 
923 Eccles, R.L. et al. 2016. Bimodal antagonism of PKA signalling by ARHGAP36. Nature Communications 7: 12963. doi: https://doi.org/10.1038/ncomms12963, PMID: 27713425.

Elias, J.E. \& Gygi, S.P. 2007. Target-decoy search strategy for increased confidence in largescale protein identifications by mass spectrometry. Nature Methods 4: 207-214. doi: https://doi.org/10.1038/nmeth1019.

Elsawa, S.F. et al. 2011. GLI2 transcription factor mediates cytokine cross-talk in the tumor microenvironment. Journal of Biological Chemistry 286: 21524-21534. doi: https://doi.org/10.1074/jbc.M111.234146.

Faucherre, A. et al. 2003. Lowe syndrome protein OCRL1 interacts with Rac GTPase in the transGolgi network. Human Molecular Genetics

12: 2449-2456. doi: https://doi.org/10.1093/hmg/ddg250.

Flora, A., Klisch, T.J., Schuster, G. \& Zoghbi, H.Y. 2009. Deletion of Atoh1 disrupts Sonic Hedgehog signaling in the developing cerebellum and prevents medulloblastoma. Science 326: 1424-1427. doi: https://doi.org/10.1126/science.1181453, PMID: 19965762.

Garcia-Gonzalo, F.R. et al. 2015. Phosphoinositides regulate ciliary protein trafficking to modulate Hedgehog signaling. Developmental Cell 34: 400-409. doi: https://doi.org/10.1016/j.devcel.2015.08.001.

Han, B. et al. 2015. FOXC1 activates Smoothened-independent Hedgehog signaling in basallike breast cancer. Cell Reports 13: 1046-1058. doi: protein Polaris for processing and function. PLoS Genetics 1: e53. doi: https://doi.org/10.1371/journal.pgen.0010053. 
946 Heldwein, E.E. et al. 2004. Crystal structure of the clathrin adaptor protein 1 core. Proceedings of the National Academy of Sciences of the United States of America 101: 14108-14113. doi: https://doi.org/10.1073/pnas.0406102101.

949

950

951

952

953

954

955

956

957

958

959

960

961

962

963

964

965

966

967

968

969

Hill, P., Götz, K. \& Rüther, U. 2009. A SHH-independent regulation of Gli3 is a significant determinant of anteroposterior patterning of the limb bud. Developmental Biology 328: 506-516. doi: https://doi.org/10.1016/j.ydbio.2009.02.017.

Hsu, J. et al. 2019. E2F4 regulates transcriptional activation in mouse embryonic stem cells independently of the RB family. Nature Communications 10: 2939. doi: https://doi.org/10.1038/s41467-019-10901-x.

Huangfu, D. \& Anderson, K.V. 2005. Cilia and Hedgehog responsiveness in the mouse. Proceedings of the National Academy of Sciences of the United States of America 102: 11325-11330. doi: https://doi.org/10.1073/pnas.0505328102.

Hui, C.-c. \& Angers, S. 2011. Gli proteins in development and disease. Annual Review of Cell and Developmental Biology 27: 513-537. doi: https://doi.org/10.1146/annurev-cellbio092910-154048, PMID: 21801010.

Humke, E.W., Dorn, K.V., Milenkovic, L., Scott, M.P. \& Rohatgi, R. 2010. The output of Hedgehog signaling is controlled by the dynamic association between Suppressor of Fused and the Gli proteins. Genes \& Development 24: 670-682. doi: https://doi.org/10.1101/gad.1902910, PMID: 20360384.

Huttlin, E.L. et al. 2017. Architecture of the human interactome defines protein communities and disease networks. Nature 545: 505-509. doi: https://doi.org/10.1038/nature22366, PMID: 28514442.

Hyman, J.M. et al. 2009. Small-molecule inhibitors reveal multiple strategies for Hedgehog pathway blockade. Proceedings of the National Academy of Sciences of the United States 
of America 106: 14132-14137. doi: https://doi.org/10.1073/pnas.0907134106, PMID: 19666565.

972

973

Incardona, J.P. et al. 2000. Receptor-mediated endocytosis of soluble and membrane-tethered sonic hedgehog by patched-1. Proceedings of the National Academy of Sciences of the United States of America 97: 12044-12049. doi: https://doi.org/10.1073/pnas.220251997.

Jaeken, J. et al. 2006. Deletion of PREPL, a gene encoding a putative serine oligopeptidase, in patients with hypotonia-cystinuria syndrome. American Journal of Human Genetics 78: 38-51. doi: https://doi.org/10.1086/498852, PMID: 16385448.

Kanie, T. et al. 2017. The CEP19-RABL2 GTPase complex binds IFT-B to linitiate intraflagellar transport at the ciliary base. Developmental Cell 42: 22-36.e12. doi: https://doi.org/10.1016/j.devcel.2017.05.016.

Kasper, M. et al. 2006. Selective modulation of Hedgehog/GLI target gene expression by epidermal growth factor signaling in human keratinocytes. Molecular and Cellular Biology 26: 6283-6298. doi: https://doi.org/10.1128/mcb.02317-05.

Kim, J., Kato, M. \& Beachy, P.A. 2009. Gli2 trafficking links Hedgehog-dependent activation of Smoothened in the primary cilium to transcriptional activation in the nucleus. Proceedings of the National Academy of Sciences of the United States of America 106: 21666-21671. doi: https://doi.org/10.1073/pnas.0912180106, PMID: 19996169.

Langmead, B. \& Salzberg, S.L. 2012. Fast gapped-read alignment with Bowtie 2. Nature Methods 9: 357-359. doi: https://doi.org/10.1038/nmeth.1923, PMID: 22388286.

Lee, Y.s. et al. 2019. A computational framework for genome-wide characterization of the human disease landscape. Cell Systems 8: 152-162. doi: https://doi.org/10.1016/j.cels.2018.12.010.

Lewis, P.M., Gritli-Linde, A., Smeyne, R., Kottmann, A. \& McMahon, A.P. 2004. Sonic hedgehog signaling is required for expansion of granule neuron precursors and patterning of the 
mouse cerebellum. Developmental Biology 270: 393-410. doi: https://doi.org/10.1016/j.ydbio.2004.03.007, PMID: 15183722.

Li, B. et al. 2017. Drebrin restricts rotavirus entry by inhibiting dynamin-mediated endocytosis. E3642-E3651. doi: https://doi.org/10.1073/pnas.1619266114.

Li, H. et al. 2009. The Sequence Alignment/Map format and SAMtools. Bioinformatics 25: 20782079. doi: https://doi.org/10.1093/bioinformatics/btp352, PMID: 19505943.

Lignitto, L. et al. 2013. Proteolysis of MOB1 by the ubiquitin ligase praja2 attenuates Hippo signalling and supports glioblastoma growth. Nature Communications 4: 1822. doi: https://doi.org/10.1038/ncomms2791.

Lignitto, L. et al. 2011. Control of PKA stability and signalling by the RING ligase praja2. Nature Cell Biology 13: 412-422. doi: https://doi.org/10.1038/ncb2209.

Liu, A., Wang, B. \& Niswander, L.A. 2005. Mouse intraflagellar transport proteins regulate both 1008 the activator and repressor functions of Gli transcription factors. Development 132: 31033111. doi: https://doi.org/10.1242/dev.01894. by stabilizing GLI2 transcription factor and inhibiting ubiquitination. Oncogene 33: 65-73. doi: https://doi.org/10.1038/onc.2012.544, PMID: 23208494.

Long, J. et al. 2014. The BET bromodomain inhibitor I-BET151 acts downstream of smoothened

Marchesi, S. et al. 2014. DEPDC1B coordinates de-adhesion events and cell-cycle progression at mitosis. Developmental Cell 31: 420-433. doi: $1018 \quad$ https://doi.org/10.1016/j.devcel.2014.09.009. 
1019 May, S.R. et al. 2005. Loss of the retrograde motor for IFT disrupts localization of Smo to cilia

1020

1021

1022

1023

1024

1025

1026

1027

1028

1029

1030

1031

1032

1033

1034

1035

1036

1037

1038

1039

1040

1041

1042

and prevents the expression of both activator and repressor functions of Gli.

Developmental Biology 287: 378-389. doi: https://doi.org/10.1016/j.ydbio.2005.08.050,

PMID: 16229832.

Moon, S. 2003. Rho GTPase-activating proteins in cell regulation. Trends in Cell Biology 13: 1322. doi: https://doi.org/10.1016/S0962-8924(02)00004-1.

Moore, B.S. et al. 2016. Cilia have high cAMP levels that are inhibited by Sonic Hedgehogregulated calcium dynamics. Proceedings of the National Academy of Sciences of the United States of America 113: 13069-13074. doi: https://doi.org/10.1073/pnas.1602393113, PMID: 27799542.

Mukhopadhyay, S. et al. 2013. The ciliary G-protein-coupled receptor Gpr161 negatively regulates the Sonic hedgehog pathway via cAMP signaling. Cell 152: 210-223. doi: https://doi.org/10.1016/j.cell.2012.12.026, PMID: 23332756.

Müller, P.M. et al. 2020. Systems analysis of RhoGEF and RhoGAP regulatory proteins reveals spatially organized RAC1 signalling from integrin adhesions. Nature Cell Biology 22: 498511. doi: https://doi.org/10.1038/s41556-020-0488-x, PMID: 32203420.

Murone, M., Rosenthal, A. \& De Sauvage, F.J. 1999. Sonic hedgehog signaling by the patchedsmoothened receptor complex. Current Biology 9: 76-84. doi: https://doi.org/10.1016/S0960-9822(99)80018-9, PMID: 10021362.

Nam, H. et al. 2019. Critical roles of ARHGAP36 as a signal transduction mediator of shh pathway in lateral motor columnar specification. elife 8: e46683. doi: https://doi.org/10.7554/eLife.46683.

Pan, Y., Bai, C.B., Joyner, A.L. \& Wang, B. 2006. Sonic hedgehog signaling regulates Gli2 transcriptional activity by suppressing its processing and degradation. Molecular and 
1043

1044

1045

1046

1047

1048

1049

1050

1051

1052

1053

1054

1055

1056

1057

1058

1059

1060

1061

1062

1063

1064

1065

1066

1067

Cellular Biology 26: 3365-3377. doi: https://doi.org/10.1128/MCB.26.9.3365-3377.2006, PMID: 16611981.

Pan, Y. \& Wang, B. 2007. A novel protein-processing domain in Gli2 and Gli3 differentially blocks complete protein degradation by the proteasome. The Journal of Biological Chemistry 282: 10846-10852. doi: https://doi.org/10.1074/jbc.M608599200, PMID: 17283082.

Pedersen, L.B., Mogensen, J.B. \& Christensen, S.T. 2016. Endocytic control of cellular signaling at the primary cilium. Trends in Biochemical Sciences 41: 787-797. doi: https://doi.org/10.1016/j.tibs.2016.06.002.

Pusapati, G.V. et al. 2018. CRISPR screens uncover genes that regulate target cell sensitivity to the morphogen Sonic Hedgehog. Developmental Cell 44: 113-129.e118. doi: https://doi.org/10.1016/j.devcel.2017.12.003.

Rack, P.G. et al. 2014. Arhgap36-dependent activation of Gli transcription factors. Proceedings of the National Academy of Sciences of the United States of America 111: 11061-11066. doi: https://doi.org/10.1073/pnas.1322362111, PMID: 25024229.

Radhakrishnan, K., Baltes, J., Creemers, J.W.M. \& Schu, P. 2013. Trans-Golgi network morphology and sorting is regulated by prolyl-oligopeptidase-like protein PREPL and the AP-1 complex subunit $\mu 1 \mathrm{~A}$. Journal of Cell Science 126: 1155-1163. doi: https://doi.org/10.1242/jcs.116079.

Riobó, N.A., Lu, K., Ai, X., Haines, G.M. \& Emerson, C.P. 2006. Phosphoinositide 3-kinase and Akt are essential for Sonic Hedgehog signaling. Proceedings of the National Academy of Sciences of the United States of America 103: 4505-4510. doi: https://doi.org/10.1073/pnas.0504337103, PMID: 16537363.

Rohatgi, R., Milenkovic, L. \& Scott, M.P. 2007. Patched1 regulates hedgehog signaling at the primary cilium. Science 317: 372-376. doi: https://doi.org/10.1126/science.1139740, PMID: 17641202. 
1068 Scheffzek, K., Ahmadian, M.R. \& Wittinghofer, A. 1998. GTPase-activating proteins: helping 1069 1070 hands to complement an active site. Trends in Biochemical Sciences 23: 257-262. doi: https://doi.org/10.1016/S0968-0004(98)01224-9.

Schindelin, J. et al. 2012. Fiji: An open-source platform for biological-image analysis. Nature

\section{3}

1074

1075

1076

1077

1078

1079

1080

1081

1082

1083

1084

1085

1086

1087

1088

1089

1090

1091

1092

Methods 9: 676-682. doi: https://doi.org/10.1038/nmeth.2019, PMID: 22743772.

Schulz, I. et al. 2002. Modulation of inositol 1,4,5-triphosphate concentration by prolyl endopeptidase inhibition. European Journal of Biochemistry 269: 5813-5820. doi: https://doi.org/10.1046/j.1432-1033.2002.03297.x, PMID: 12444969.

Sepe, M. et al. 2014. Proteolytic control of neurite outgrowth inhibitor NOGO-A by the cAMP/PKA pathway. Proceedings of the National Academy of Sciences of the United States of America 111: 15729-15734. doi: https://doi.org/10.1073/pnas.1410274111.

Stamataki, D., Ulloa, F., Tsoni, S.V., Mynett, A. \& Briscoe, J. 2005. A gradient of Gli activity mediates graded Sonic Hedgehog signaling in the neural tube. Genes \& Development 19: 626-641. doi: https://doi.org/10.1101/gad.325905.

Stone, D.M. et al. 1996. The tumour-suppressor gene patched encodes a candidate receptor for Sonic hedgehog. Nature 384: 129-134. doi: https://doi.org/10.1038/384129a0.

Stone, D.M. et al. 1999. Characterization of the human Suppressor of fused a negative regulator of the zinc-finger transcription factor Gli. Journal of Cell Science 112: 4437-4448. PMID: 10564661.

Szeltner, Z., Alshafee, I., Juhász, T., Parvari, R. \& Polgár, L. 2005. The PREPL A protein, a new member of the prolyl oligopeptidase family, lacking catalytic activity. Cellular and Molecular Life Sciences 62: 2376-2381. doi: https://doi.org/10.1007/s00018-005-5262-5.

Taipale, J. et al. 2000. Effects of oncogenic mutations in Smoothened and Patched can be reversed by cyclopamine. Nature 406: 1005-1009. doi: https://doi.org/10.1038/35023008, PMID: 10984056. 
1093 Taipale, J., Cooper, M.K., Maiti, T. \& Beachy, P.A. 2002. Patched acts catalytically to suppress

1094

1095

1096

1097

1098

1099

1100

1101

1102

1103

1104

1105

1106

1107

1108

1109

1110

1111

1112

1113

1114

1115

1116

1117

the activity of smoothened. Nature

418: $\quad 892-897$.

doi: https://doi.org/10.1038/nature00989, PMID: 12192414.

te Welscher, P. et al. 2002. Progression of vertebrate limb development through SHH-mediated counteraction $\quad$ of

of GLI3.

Science

298: 827-830.

doi: https://doi.org/10.1126/science.1075620.

Tempe, D., Casas, M., Karaz, S., Blanchet-Tournier, M.-F. \& Concordet, J.-P. 2006. Multisite protein kinase $A$ and glycogen synthase kinase 3 phosphorylation leads to Gli3 ubiquitination by SCF $\beta-T r C P$. Molecular and Cellular Biology 26: 4316-4326. doi: https://doi.org/10.1128/mcb.02183-05.

Torres, J.Z., Miller, J.J. \& Jackson, P.K. 2009. High-throughput generation of tagged stable cell lines for proteomic analysis. Proteomics 9: 2888-2891. doi: https://doi.org/10.1002/pmic.200800873.

Tukachinsky, H., Lopez, L.V. \& Salic, A. 2010. A mechanism for vertebrate Hedgehog signaling: recruitment to cilia and dissociation of SuFu-Gli protein complexes. The Journal of Cell Biology 191: 415-428. doi: https://doi.org/10.1083/jcb.201004108, PMID: 20956384.

Tuson, M. et al. 2011. Protein kinase A acts at the basal body of the primary cilium to prevent Gli2 activation and ventralization of the mouse neural tube. Development 138: 4921-4930. doi: https://doi.org/10.1242/dev.070805, PMID: 22007132.

Vuolo, L., Herrera, A., Torroba, B., Menendez, A. \& Pons, S. 2015. Ciliary adenylyl cyclases control the Hedgehog pathway. Journal of Cell Science 128: 2928-2937. doi: https://doi.org/10.1242/jcs.172635.

Wallace, V.A. 1999. Purkinje-cell-derived Sonic hedgehog regulates granule neuron precursor cell proliferation in the developing mouse cerebellum. Current Biology 9: 445-448. doi: https://doi.org/10.1016/S0960-9822(99)80195-X. 
1118 Wang, B. \& Li, Y. 2006. Evidence for the direct involvement of $\beta$-TrCP in Gli3 protein processing.

1119

1120

1121

1122

1123

1124

1125

1126

1127

1128

1129

1130

1131

1132

1133

1134

1135

1136

1137

1138

1139

1140 Proceedings of the National Academy of Sciences of the United States of America 103: 33-38. doi: https://doi.org/10.1073/pnas.0509927103.

Wang, C., Pan, Y. \& Wang, B. 2010. Suppressor of fused and Spop regulate the stability processing and function of Gli2 and Gli3 full-length activators but not their repressors. Development 137: 2001-2009. doi: https://doi.org/10.1242/dev.052126, PMID: 20463034.

Wang, Y., Zhou, Z., Walsh, C.T. \& McMahon, A.P. 2009. Selective translocation of intracellular Smoothened to the primary cilium in response to Hedgehog pathway modulation. Proceedings of the National Academy of Sciences of the United States of America 106: 2623-2628. doi: https://doi.org/10.1073/pnas.0812110106.

Wang, Y.J. et al. 2003. Phosphatidylinositol 4 phosphate regulates targeting of clathrin adaptor AP-1 complexes to the Golgi. Cell 114: 299-310. doi: https://doi.org/10.1016/S00928674(03)00603-2, PMID: 12914695.

Wechsler-Reya, R. \& Scott, M.P. 2001. The developmental biology of brain tumors. Annual Review of Neuroscience

24: $385-428$.

doi: https://doi.org/10.1146/annurev.neuro.24.1.385, PMID: 11283316.

Wen, X. et al. 2010. Kinetics of Hedgehog-dependent full-length Gli3 accumulation in primary cilia and subsequent degradation. Molecular and Cellular Biology 30: 1910-1922. doi: https://doi.org/10.1128/mcb.01089-09.

Williams, R.S.B., Eames, M., Ryves, W.J., Viggars, J. \& Harwood, A.J. 1999. Loss of a prolyl oligopeptidase confers resistance to lithium by elevation of inositol $(1,4,5)$ trisphosphate. EMBO Journal 18: 2734-2745. doi: https://doi.org/10.1093/emboj/18.10.2734, PMID: 10329620. 
1141 Wright, K.J. et al. 2011. An ARL3-UNC119-RP2 GTPase cycle targets myristoylated NPHP3 to

1142 the primary cilium. Genes and Development 25: 2347-2360. doi:

1143 https://doi.org/10.1101/gad.173443.111, PMID: 22085962.

1144 Zhang, B. et al. 2019. Patched1-ArhGAP36-PKA-Inversin axis determines the ciliary 1145 translocation of Smoothened for Sonic Hedgehog pathway activation. Proceedings of the $1146 \quad$ National Academy of Sciences of the United States of America 116: 874-879. doi: 1147 https://doi.org/10.1073/pnas.1804042116, PMID: 30598432. 
bioRxiv preprint doi: https://doi.org/10.1101/2020.05.14.094961; this version posted May 24, 2020. The copyright holder for this preprint (which was not certified by peer review) is the author/funder. All rights reserved. No reuse allowed without permission.
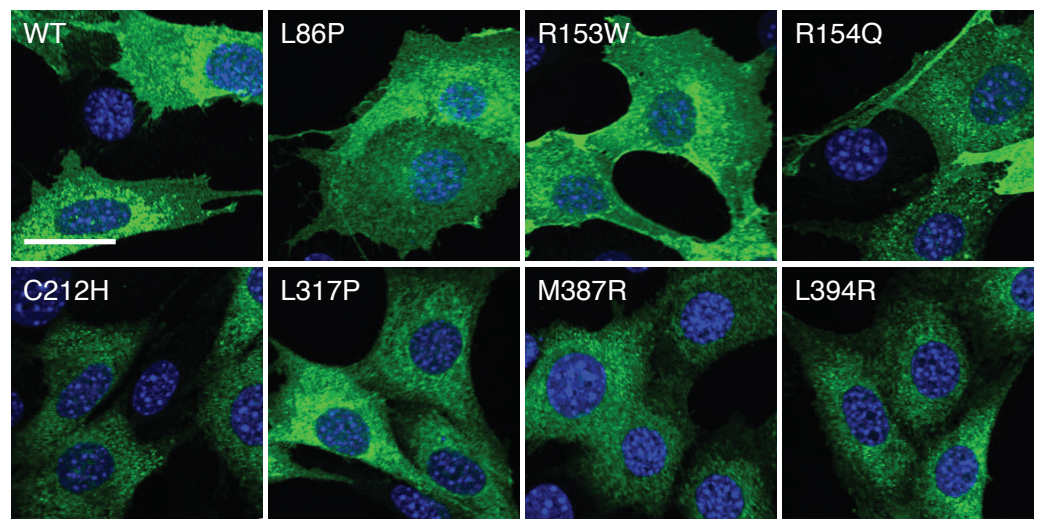

mCherry DAPI

Figure 4 - figure supplement 1. Subcellular localization of ARHGAP36 isoform 2 point mutants. Representative immunofluorescence micrographs of SHH-EGFP cells retrovirally transduced with the indicated mCherry-tagged ARHGAP36 constructs. Scale bar: $20 \mu \mathrm{m}$. 
bioRxiv preprint doi: https://doi.org/10.1101/2020.05.14.094961; this version posted May 24, 2020. The copyright holder for this preprint (which was not certified by peer review) is the author/funder. All rights reserved. No reuse allowed without permission.

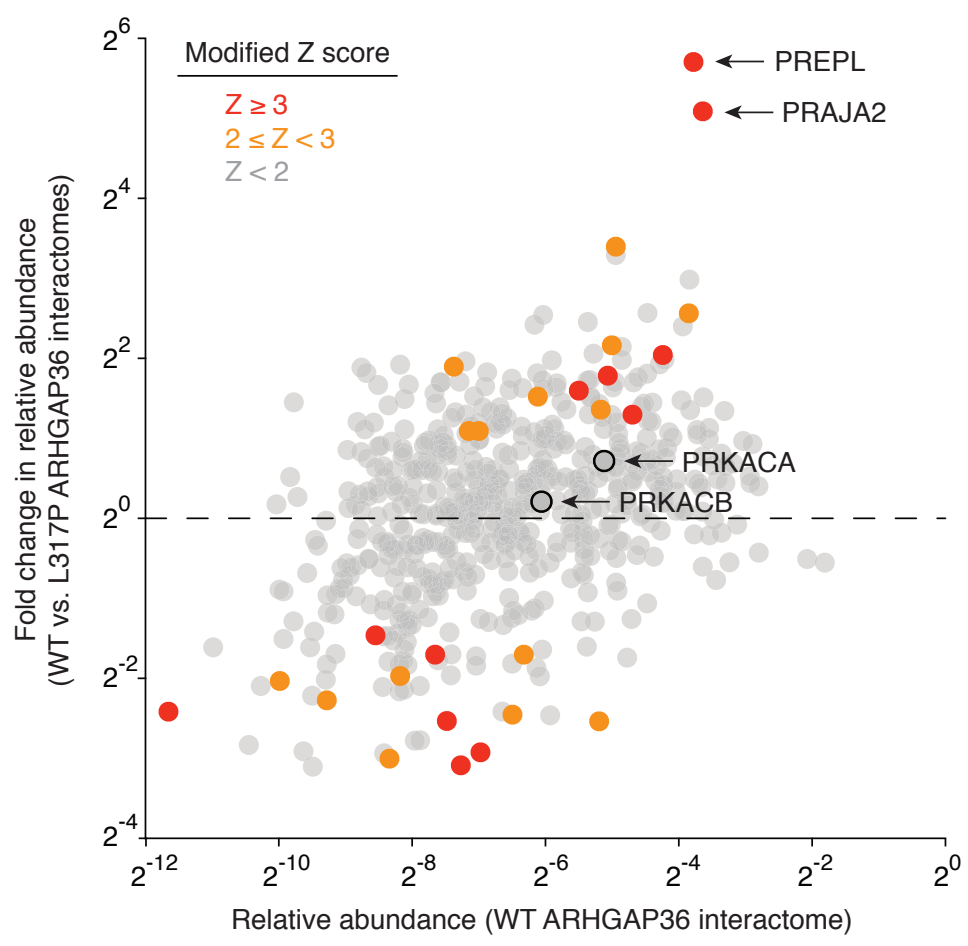

Figure 6 - figure supplement 1. Comparative analyses of the wild-type and L317P ARHGAP36 interactomes. Scatter plot of ARHGAP36 isoform 2-binding proteins according to their normalized spectral abundance in the wild-type and L317P interactomes. Each data point represents the average value for three biological replicates, and a modified Z-score was calculated for each fold change in abundance (see Methods). 